\title{
OPTIMAL LOCATION OF THE SUPPORT OF THE CONTROL FOR THE 1-D WAVE EQUATION: NUMERICAL INVESTIGATIONS
}

\author{
Arnaud Münch * \\ March 22, 2006
}

\begin{abstract}
We consider in this paper the homogeneous 1-D wave equation defined on $\Omega \subset \mathbb{R}$. Using the Hilbert Uniqueness Method, one may define, for each subset $\omega \subset \Omega$, the exact control $v_{\omega}$ of minimal $L^{2}(\omega \times(0, T))$-norm which drives to rest the system at a large enough time $T>0$. We address the question of the optimal position of $\omega$ which minimizes the functional $J: \omega \rightarrow\left\|v_{\omega}\right\|_{L^{2}(\omega \times(0, T))}$. We express the shape derivative of $J$ as an integral on $\partial \omega \times(0, T)$ independently of any adjoint solution. This expression leads to a descent direction for $J$ and permits to define a gradient algorithm efficiently initialized by the topological derivative associated to $J$. The numerical approximation of the problem is discussed and numerical experiments are presented in the framework of the level set approach. We also investigate the well-posedness character of the problem by considering its convexification.
\end{abstract}

Key Words. Optimal shape design, exact controllability, wave equation, level set method, numerical schemes, convexification.

Mathematics Subject Classification. 35L05, 49J20, 65K10, 65M60, 93B05.

\section{Introduction - Problem statement}

Let us consider a bounded domain $\Omega \in \mathbb{R}$ and a subset $\omega \subset \Omega$ of positive Lebesgue measure $|\omega|$ which may be composed of a finite number of disjoints components. In the context of the exact controllability for the 1-D homogeneous wave equation, the following result is well-known ([13], Theorem 2.6, page 423): for any time $T>$ $T^{\star}(\Omega \backslash \omega)\left(T^{\star}(\Omega \backslash \omega)\right.$ depends on the diameter of $\left.\Omega \backslash \omega\right)$ and any initial data $\left(y^{0}, y^{1}\right) \in$ $H_{0}^{1}(\Omega) \times L^{2}(\Omega)$ independent of $\omega$, there exists a control function $v_{\omega} \in L^{2}(\omega \times(0, T))$ such that the unique solution $y \in C\left([0, T] ; H_{0}^{1}(\Omega)\right) \cap C^{1}\left([0, T] ; L^{2}(\Omega)\right)$ of

$$
\begin{cases}y_{t t}-\Delta y=v_{\omega} \mathcal{X}_{\omega}, & \Omega \times(0, T), \\ y=0, & \partial \Omega \times(0, T), \\ \left(y(\cdot, 0), y_{t}(\cdot, 0)\right)=\left(y^{0}, y^{1}\right), & \Omega,\end{cases}
$$

${ }^{*}$ Laboratoire de Mathématiques de Besançon, UMR CNRS 6623, Université de Franche-Comte, 16 route de Gray 25030 Besançon cedex, France, (arnaud.munch@math.univ-fcomte.fr). 
satisfies

$$
y(., T)=y_{t}(., T)=0, \quad \text { in } \quad \Omega .
$$

$y_{t}$ denotes the derivative of $y$ with respect to $t$ and $\mathcal{X}_{\omega} \in L^{\infty}(\Omega,\{0,1\})$ denotes the characteristic function of the subset $\omega$.

The control problem formulated above is usually referred to as internal (or distributed) controllability problem. The controllability property may be obtained using the Hilbert Uniqueness Method (HUM) introduced by J.-L. Lions in [13], which reduces the problem to an optimal control one. Precisely, the unique control $v_{\omega}$ of minimal $L^{2}$-norm (refereed as the HUM control in the sequel) may be obtained by minimizing the functional $\mathcal{J}: L^{2}(\Omega) \times H^{-1}(\Omega) \rightarrow \mathbb{R}$ defined by

$$
\mathcal{J}\left(\phi^{0}, \phi^{1}\right)=\frac{1}{2} \int_{\omega} \int_{0}^{T} \phi^{2} d t d x+<\phi_{t}(\cdot, 0), y^{0}>_{H^{-1}(\Omega), H_{0}^{1}(\Omega)}-\int_{\Omega} y^{1} \phi(\cdot, 0) d x
$$

where $\langle\cdot, \cdot\rangle_{H^{-1}, H_{0}^{1}}$ denotes the duality product between $H^{-1}(\Omega)$ and $H_{0}^{1}(\Omega)$ and $\phi$ the solution of the adjoint homogeneous system

$$
\begin{cases}\phi_{t t}-\Delta \phi=0, & \Omega \times(0, T), \\ \phi=0, & \partial \Omega \times(0, T), \\ \left(\phi(\cdot, 0), \phi_{t}(\cdot, 0)\right)=\left(\phi^{0}, \phi^{1}\right), & \Omega .\end{cases}
$$

This provides the following characterization of the HUM-control (see [13], chapter 7).

Theorem 1.1 Given any $\omega \in \Omega, T>T^{\star}(\Omega \backslash \omega)$ and $\left(y^{0}, y^{1}\right) \in H_{0}^{1}(\Omega) \times L^{2}(\Omega)$ the functional $\mathcal{J}$ has a unique minimizer $\left(\phi_{0}, \phi_{1}\right) \in L^{2}(\Omega) \times H^{-1}(\Omega)$. If $\phi$ is the corresponding solution of (4) with initial data $\left(\phi^{0}, \phi^{1}\right)$ then $v=-\phi \mathcal{X}_{\omega}$ is the control of (1) with minimal $L^{2}$-norm.

This result is based on the following observation or observability inequality (leading to the coercivity of $\mathcal{J}$ in $L^{2}(\Omega) \times H^{-1}(\Omega)$ ): there exists a constant $C_{T}>0$ function of $T$ (called the observability constant) such that

$$
\left\|\left(\phi^{0}, \phi^{1}\right)\right\|_{L^{2}(\Omega) \times H^{-1}(\Omega)}^{2} \leq C_{T} \int_{\omega} \int_{0}^{T} \phi^{2}(\boldsymbol{x}, t) d t d x
$$

for all $\left(\phi^{0}, \phi^{1}\right) \in L^{2}(\Omega) \times H^{-1}(\Omega)$. Therefore, from a practical viewpoint, $\omega$ being fixed, such a control is determined by solving the linear problem

$$
\Lambda\left(\phi^{0}, \phi^{1}\right)=\left(y^{1},-y^{0}\right) \quad \text { in } \Omega,
$$

where the isomorphism $\Lambda$ from $L^{2}(\Omega) \times H^{-1}(\Omega)$ onto $H_{0}^{1}(\Omega) \times L^{2}(\Omega)$ is defined by $\Lambda\left(\phi^{0}, \phi^{1}\right):=\left(\psi_{t}(0),-\psi(0)\right)$ with $\psi$ the unique solution of

$$
\begin{cases}\psi_{t t}-\Delta \psi=-\phi \mathcal{X}_{\omega}, & \Omega \times(0, T), \\ \psi=0, & \partial \Omega \times(0, T), \\ \left(\psi(\cdot, T), \psi_{t}(\cdot, T)\right)=(0,0), & \Omega .\end{cases}
$$

The HUM control is then $v_{\omega}=-\phi \mathcal{X}_{\omega}$ and $y=\psi$. 
Related to the controllability problem (1)-(2), we consider for any real $L \in(0,1)$, the following problem :

$$
\left(\mathcal{P}_{\omega}\right): \inf _{\omega \subset \Omega_{L}} J\left(\mathcal{X}_{\omega}\right), \quad \text { where } J\left(\mathcal{X}_{\omega}\right)=\frac{1}{2}\left\|v_{\omega}\right\|_{L^{2}(\omega \times(0, T))}^{2},
$$

and $\Omega_{L}=\{\omega \in \Omega ;|\omega| \leq L|\Omega|\}$ which consists in finding the optimal location of $\omega \in \Omega_{L}$ in order to minimize the $L^{2}$-norm of the corresponding HUM-control $v_{\omega}$. The size restriction on $\omega$ will be justified in the sequel. This optimal location problem may be not well-posed in the sense of no existence of solution in the class of characteristic function: the optimal subset $\omega$ may be composed of an arbitrarily large number of disjoints components. A sufficient condition for $\left(\mathcal{P}_{\omega}\right)$ to be well-posed is to restrict the number of disjoints components (we refer to the books [8] and [12] for a complete description in the static case).

In this work, we numerically solve the problem $\left(\mathcal{P}_{\omega}\right)$ and proceed as in [15] where the author considers a damped wave equation and optimizes the position of the damping zone in order to minimize the energy of the system at a given time $T>0$. Since the control associated to the optimal solution $\omega$ is a fortiori a HUM control (that is of minimal $L^{2}(0, T)$ norm $),\left(\mathcal{P}_{\omega}\right)$ is reduced to find the optimal HUM control with respect to $\omega$. In this way, we make use of the explicit characterization of $v_{\omega}$ in term of the solution $\phi$ of (4). To the knowledge of the author, the problem $\left(\mathcal{P}_{\omega}\right)$ has never been studied so far. In the similar context of the boundary controllability, it is worth to mention the work of Asch-Lebeau [2] where the relationships between the geometry of the domain, the geometry of the controlled boundary, and the energy of the control are dealt with. Moreover, in order to take into account the size restriction, we introduce a positive multiplier $\lambda$ and then consider the problem :

$$
\inf _{\omega \subset \Omega, \lambda \in \mathbb{R}^{+}} J_{\lambda}\left(\mathcal{X}_{\omega}\right) \quad \text { where } \quad J_{\lambda}\left(\mathcal{X}_{\omega}\right)=J\left(\mathcal{X}_{\omega}\right)+\lambda\left\|\mathcal{X}_{\omega}\right\|_{L^{1}(\Omega)}
$$

The outline of the paper is as follows. In section 2, we first compute the variation of $J_{\lambda}$ with respect to smooth variations of $\omega$. This derivative expressed as a pointwise integral on $\partial \omega \times(0, T)$ independently of any adjoint solution permits to define a descent direction and build a minimizing sequence of domains for $J_{\lambda}$. The corresponding gradient algorithm is presented in section 3 in the framework of the level set approach and is efficiently initialized by the topological derivative associated to $J_{\lambda}$. In section 4 , we point out the sensibility of the controllability problem with respect to the numerical approximation, and then present an efficient and robust semi-discrete scheme in space to solve (6) (the resolution (analytical in time) is discussed in the appendix 8.1). In section 5 , simple numerical experiments are detailed and discussed. Then, in section 6 , we investigate whether or not the problem $\left(\mathcal{P}_{\omega}\right)$ is well-posed by considering its convexification $(\mathcal{C P} \omega)$. Numerical examples are given and compared with those of section 5. Finally, we give some concluding remarks and perspectives in section 7 .

\section{Shape and topological derivatives of $J_{\lambda}$}

In order to solve the problem $\left(\mathcal{P}_{\omega}\right)$ with a gradient descent procedure, we compute explicitly an expression of the derivative of the functional $J_{\lambda}$ with respect to smooth variations of $\omega$ (the so-called shape derivative). Let $\eta \in \mathbb{R}^{+}$and a vector field $\boldsymbol{\theta} \in$ 
$W^{1, \infty}(\Omega, \mathbb{R}), \boldsymbol{\theta}_{\mid \partial \Omega}=0$ and not vanishing on a neighborhood of $\partial \omega$. Smooth perturbation $\omega^{\eta}$ of $\omega$ are then defined by the transformation $\mathcal{F}^{\eta}: \boldsymbol{x} \rightarrow \boldsymbol{x}+\eta \boldsymbol{\theta}(\boldsymbol{x}) \in \Omega^{\eta}=\Omega$ such that $\omega^{\eta}=\mathcal{F}^{\eta}(\omega)$. The parameter $\eta$ is arbitrarily small so that $\operatorname{det}\left(\nabla \mathcal{F}^{\eta}\right)>0$ in $\Omega$ and $\omega^{\eta} \subset \Omega$.

Definition 2.1 The derivative of the functional $J_{\lambda}$ with respect to a variation of $\omega \subset \Omega$ in the direction $\boldsymbol{\theta}$ is defined as the Fréchet derivative in $W^{1, \infty}(\Omega, \mathbb{R})$ at 0 of the application $\eta \rightarrow J_{\lambda}\left(\mathcal{X}_{(I d+\eta \boldsymbol{\theta})(\omega)}\right)$, i.e.

$$
J_{\lambda}\left(\mathcal{X}_{(I d+\eta \boldsymbol{\theta})(\omega)}\right)=J_{\lambda}\left(\mathcal{X}_{\omega}\right)+\eta \frac{\partial J_{\lambda}\left(\mathcal{X}_{\omega}\right)}{\partial \omega} \cdot \boldsymbol{\theta}+o(\eta) .
$$

It is worth to mention that the initial condition $\left(y^{0}, y^{1}\right)$ is independent of $\omega$ and that the field $\boldsymbol{\theta}$ is time independent (since $\omega$ is time independent). We refer the reader to $[8]$ for the methodology to compute the shape derivative. We obtain the following result.

THEOREM 2.2 Let $T>T^{\star}(\Omega \backslash \omega)$ and $v_{\omega}$ the HUM control for (1). If $\left(y^{0}, y^{1}\right) \in$ $\left(H^{2}(\Omega) \cap H_{0}^{1}(\Omega)\right) \times H_{0}^{1}(\Omega)$ then the first lagrangian derivative of $J_{\lambda}$ with respect to $\omega$ exists and is given by the following expression:

$$
\frac{\partial J_{\lambda}\left(\mathcal{X}_{\omega}\right)}{\partial \omega} \cdot \boldsymbol{\theta}=\frac{1}{2} \int_{\omega} \int_{0}^{T}\left(2 v_{\omega} V_{\omega}+v_{\omega}^{2} \operatorname{div} \boldsymbol{\theta}\right) d t d x+\lambda \int_{\omega} \operatorname{div} \boldsymbol{\theta} d x
$$

where $V_{\omega}$ is the control of minimal $L^{2}(\omega \times(0, T))$-norm associated to the following system:

$$
\begin{cases}Y_{t t}-\Delta Y-\Delta \boldsymbol{\theta} \nabla y+2 \operatorname{div}(\nabla \boldsymbol{\theta} \nabla y)=V_{\omega} \mathcal{X}_{\omega}, & \Omega \times(0, T), \\ Y=0, & \partial \Omega \times(0, T), \\ \left(Y(\cdot, 0), Y_{t}(\cdot, 0)\right)=\left(\nabla y^{0} \cdot \boldsymbol{\theta}, \nabla y^{1} \cdot \boldsymbol{\theta}\right), & \Omega,\end{cases}
$$

such that $Y(\cdot, T)=Y_{t}(\cdot, T)=0$ in $\Omega$.

A sketch of the technical proof is presented in the appendix 8.1. The couple $\left(Y, V_{\omega}\right)$ is the first lagrangian derivative of $\left(y, v_{\omega}\right)$ and is as expected the solution of an exact controllability problem. Remark that the null controllability of (12) may be obtained from the Hilbert Uniqueness Method (see Theorem 2.6 page 423) since $\left(Y(\cdot, 0), Y_{t}(\cdot, 0)\right) \in H_{0}^{1}(\Omega) \times L^{2}(\Omega)$.

Moreover, as well known, the shape derivative may be expressed in terms of a pointwise integral on $\partial \omega$. We obtain the following result.

Theorem 2.3 Let $T>T^{\star}(\Omega \backslash \omega)$, $v_{\omega}$ the HUM control for (1) and $\boldsymbol{\nu}$ be the unit normal vector oriented toward the exterior of $\omega$. If $\left(y^{0}, y^{1}\right) \in\left(H^{2}(\Omega) \cap H_{0}^{1}(\Omega)\right) \times H_{0}^{1}(\Omega)$ then the derivative of $J_{\lambda}$ with respect to $\omega$ is given by the following expression:

$$
\frac{\partial J_{\lambda}\left(\mathcal{X}_{\omega}\right)}{\partial \omega} \cdot \boldsymbol{\theta}=-\frac{1}{2} \int_{\partial \omega} \int_{0}^{T} v_{\omega}^{2}(\boldsymbol{x}, t) d t \boldsymbol{\theta} \cdot \boldsymbol{\nu} d \sigma+\lambda \int_{\partial \omega} \boldsymbol{\theta} \cdot \boldsymbol{\nu} d \sigma .
$$


Proof of the Theorem 2.3. The expression (13) may be obtained from the relation (11) after many integrations by part with respect to $\boldsymbol{x}$ and $t$. We refer to [15] for an example of calculation in a similar context and to [8] for a general presentation. Simpler, we use the Cea's method [7] and introduce the Lagrangian

$$
\begin{aligned}
\mathcal{L}(\omega, \bar{\phi}, \bar{\psi}, \bar{p}, \bar{q})= & \frac{1}{2} \int_{\omega} \int_{0}^{T} \bar{\phi}^{2} d t d x \\
& -\int_{\Omega} \int_{0}^{T} \bar{\phi}^{\prime} \bar{p}^{\prime} d t d x+\int_{\Omega}\left[\bar{\phi}^{\prime} \bar{p}\right]_{0}^{T} d x+\int_{\Omega} \int_{0}^{T} \nabla \bar{\phi} \cdot \nabla \bar{p} d t d x \\
& -\int_{\Omega} \int_{0}^{T} \bar{\psi}^{\prime} \bar{q}^{\prime} d t d x+\int_{\Omega}\left[\bar{\psi}^{\prime} \bar{q}\right]_{0}^{T} d x+\int_{\Omega} \int_{0}^{T} \nabla \bar{\psi} \cdot \nabla \bar{q} d t d x \\
& +\int_{\omega} \int_{0}^{T} \bar{\phi} \bar{q} d t d x,
\end{aligned}
$$

for all $\bar{\psi}, \bar{p} \in C\left([0, T] ; H^{2}(\Omega) \cap H_{0}^{1}(\Omega)\right) \cap C^{1}\left([0, T] ; H_{0}^{1}(\Omega)\right), \bar{\phi}, \bar{q} \in C\left([0, T] ; H_{0}^{1}(\Omega)\right) \cap$ $C^{1}\left([0, T] ; L^{2}(\Omega)\right) \cdot \mathcal{L}$ is chosen so that the optimality conditions $<\frac{\partial \mathcal{L}}{\partial \bar{p}}(\omega, \phi, \bar{\psi}, \bar{p}, q), p>=$ 0 and $<\frac{\partial \mathcal{L}}{\partial \bar{q}}(\omega, \bar{\phi}, \psi, p, \bar{q}), q>=0$ lead for all $p, q$ to respectively the weak formulations associated to $\phi$ and $\psi$ solutions of (4)-(6)-(7):

$$
-\int_{\Omega} \int_{0}^{T} \phi^{\prime} \bar{p}^{\prime} d t d x+\int_{\Omega}\left[\phi^{\prime} \bar{p}\right]_{0}^{T} d x+\int_{\Omega} \int_{0}^{T} \nabla \phi \cdot \nabla \bar{p} d t d x=0
$$

and

(16) $-\int_{\Omega} \int_{0}^{T} \psi^{\prime} \bar{q}^{\prime} d t d x+\int_{\Omega}\left[\psi^{\prime} \bar{q}\right]_{0}^{T} d x+\int_{\Omega} \int_{0}^{T} \nabla \psi \cdot \nabla \bar{q} d t d x=-\int_{\omega} \int_{0}^{T} \phi \bar{q} d t d x$.

We then write formally that

$$
\begin{aligned}
\frac{d \mathcal{L}}{d \omega}(\boldsymbol{\theta})= & \frac{\partial}{\partial \omega} \mathcal{L}(\omega, \bar{\phi}, \bar{\psi}, \bar{p}, \bar{q}) \cdot \boldsymbol{\theta}+<\frac{\partial}{\partial \bar{\phi}} \mathcal{L}(\omega, \bar{\phi}, \bar{\psi}, \bar{p}, \bar{q}), \frac{\partial \bar{\phi}}{\partial \omega} \cdot \boldsymbol{\theta}> \\
& +<\frac{\partial}{\partial \bar{\psi}} \mathcal{L}(\omega, \bar{\phi}, \bar{\psi}, \bar{p}, \bar{q}), \frac{\partial \bar{\psi}}{\partial \omega} \cdot \boldsymbol{\theta}>+<\frac{\partial}{\partial \bar{p}} \mathcal{L}(\omega, \bar{\phi}, \bar{\psi}, \bar{p}, \bar{q}), \frac{\partial \bar{p}}{\partial \omega} \cdot \boldsymbol{\theta}> \\
& +<\frac{\partial}{\partial \bar{q}} \mathcal{L}(\omega, \bar{\phi}, \bar{\psi}, \bar{p}, \bar{q}), \frac{\partial \bar{q}}{\partial \omega} \cdot \boldsymbol{\theta}>
\end{aligned}
$$

where $\frac{\partial \bar{p}}{\partial \boldsymbol{\omega}} \cdot \boldsymbol{\theta}$ denotes the first derivative of $\bar{p}$ in the direction $\boldsymbol{\theta}$. The variables $\bar{\phi}, \bar{\psi}, \bar{p}$ and $\bar{q}$ being independent of $\omega$, the first term in (17) is

$$
\frac{\partial}{\partial \omega} \mathcal{L}(\omega, \bar{\phi}, \bar{\psi}, \bar{p}, \bar{q})(\boldsymbol{\theta})=\frac{1}{2} \int_{\omega} \int_{0}^{T} \operatorname{div}\left(\bar{\phi}^{2} \boldsymbol{\theta}\right) d t d x+\int_{\omega} \int_{0}^{T} \operatorname{div}(\bar{\phi} \bar{p} \boldsymbol{\theta}) d t d x .
$$

In addition, by definition of $\mathcal{L}$, the fourth and fifth terms are equal to zero for $(\bar{\phi}, \bar{\psi})=$ $(\phi, \psi)$ solution of (4)-(7). Let us then determine the adjoint functions $p$ and $q$ such that the following equality

$$
\left.<\frac{\partial}{\partial \phi} \mathcal{L}(\omega, \bar{\phi}, \bar{\psi}, p, q), \frac{\partial \bar{\phi}}{\partial \omega} \cdot \boldsymbol{\theta}>+<\frac{\partial}{\partial \psi} \mathcal{L}(\omega, \bar{\phi}, \bar{\psi}, p, q), \frac{\partial \bar{\psi}}{\partial \omega} \cdot \boldsymbol{\theta}\right)>=0,
$$


holds for all $\bar{\phi} \in C\left([0, T] ; H_{0}^{1}(\Omega)\right) \cap C^{1}\left([0, T] ; L^{2}(\Omega)\right)$ and for all $\bar{\psi} \in C\left([0, T] ; H^{2}(\Omega) \cap\right.$ $\left.H_{0}^{1}(\Omega)\right) \cap C^{1}\left([0, T] ; H_{0}^{1}(\Omega)\right)$. The interpretation of (19) implies that $q$ is the unique solution in $C\left([0, T] ; H_{0}^{1}(\Omega)\right) \cap C^{1}\left([0, T] ; L^{2}(\Omega)\right)$ (the regularity of $\phi$ ) of

$$
\begin{cases}q_{t t}-\Delta q=0, & \Omega \times(0, T), \\ q=0, & \partial \Omega \times(0, T), \\ \left(q(\cdot, 0), q_{t}(\cdot, 0)\right)=\left(q^{0}, q^{1}\right), & \Omega,\end{cases}
$$

where $\left(q^{0}, q^{1}\right) \in H_{0}^{1}(\Omega) \times L^{2}(\Omega)$ is such that the solution $p \in C\left([0, T] ; H^{2}(\Omega) \cap\right.$ $\left.H_{0}^{1}(\Omega)\right) \cap C^{1}\left([0, T] ; H_{0}^{1}(\Omega)\right)$ of

$$
\begin{cases}p_{t t}-\Delta p=-(\phi+q) \mathcal{X}_{\omega} \equiv-F \mathcal{X}_{\omega}, & \Omega \times(0, T), \\ p=0, & \partial \Omega \times(0, T), \\ \left(p(\cdot, T), p_{t}(\cdot, T)\right)=(\mathbf{0}, \mathbf{0}), & \Omega,\end{cases}
$$

fulfills $\left(p(\cdot, 0), p_{t}(\cdot, 0)\right)=(\mathbf{0}, \mathbf{0}) \in\left(H^{2}(\Omega) \cap H_{0}^{1}(\Omega)\right) \times H_{0}^{1}(\Omega)$ ! Equivalently, the function $F \equiv \phi+q$ fulfills the formulation

$$
\begin{cases}F_{t t}-\Delta F=0, & \Omega \times(0, T), \\ F=0, & \partial \Omega \times(0, T), \\ \left(F(\cdot, 0), F_{t}(\cdot, 0)=\left(\phi^{0}+q^{0}, \phi^{1}+q^{1}\right),\right. & \Omega,\end{cases}
$$

where $\left(\phi^{0}+q^{0}, \phi^{1}+q^{1}\right)$ is such that $\left(p(\cdot, 0), p_{t}(\cdot, 0)\right)=(\mathbf{0}, \mathbf{0})$ in $\Omega$. The restriction $-F \mathcal{X}_{\omega}$ is then the control of minimal $L^{2}(\omega \times(0, T))$ norm which stabilizes at time $T$ the functions $\left(F(\cdot, T), F_{t}(\cdot, T)\right)$ starting from the initial state $\left(F(\cdot, 0), F_{t}(\cdot, 0)\right)=(\mathbf{0}, \mathbf{0})$ (using the same argument than $-\phi \mathcal{X}_{\omega}$ for $y$ and the reversibility in time of the system (21)). We then deduce that $F=0$ in $\omega \times(0, T)$ and then on $\Omega \times(0, T)$ (by the Holmgren Theorem, see [13], Lemma 2.5 page 423). Consequently, $q=-\phi$ on $\Omega \times(0, T)$. Then, writing $J_{0}(\omega)=\mathcal{L}(\omega, \phi, \psi, p, q)$, we get from (18),

$$
\frac{\partial J_{0}\left(\mathcal{X}_{\omega}\right)}{\partial \omega} \cdot \boldsymbol{\theta}=\frac{\partial}{\partial \omega} \mathcal{L}(\omega, \phi, \psi, p, q) \cdot \boldsymbol{\theta}=-\frac{1}{2} \int_{\omega} \int_{0}^{T} \operatorname{div}\left(\phi^{2} \boldsymbol{\theta}\right) d t d x
$$

then the relation (13) assuming enough regularity on $v_{\omega}$.

Remark 1 - As expected, the adjoint functions $(p, q)$, dual of the first lagrangian derivatives $\left(\frac{\partial \phi}{\partial \omega} \cdot \boldsymbol{\theta}, \frac{\partial \psi}{\partial \omega} \cdot \boldsymbol{\theta}\right)$ solve an exact (adjoint) control problem. Moreover, we remark that the shape derivative (13) is expressed independently of the adjoint solution $(p, q)$. This is due to the minimal $L^{2}(0, T)$-norm property of the HUM control $v_{\omega}$.

- It results from the relation (13) that the inclusion $\omega_{1} \subset \omega_{2} \subset \Omega$ implies $J_{0}\left(\omega_{2}\right) \leq$ $J_{0}\left(\omega_{1}\right)$. In particular, for $\lambda=0$, the optimal domain is $\omega=\Omega$. This justifies the introduction of the subset $\Omega_{L}$ in the formulation of $\left(\mathcal{P}_{\omega}\right)$. Similarly, for any $\omega$ fixe, the functional $J_{0}$ is a decreasing function of $T$ (see [2]).

- In order that the previous integral makes sense, it is enough that $v_{\omega} \in L^{\infty}\left(0, T, H_{0}^{1}(\Omega)\right)$ implying that $v_{\omega}^{2} \in L^{\infty}\left(0, T, W^{1,1}(\Omega)\right)$. This regularity hold if we assume that $\left(y^{0}, y^{1}\right) \in\left(H^{2}(\Omega) \cap H_{0}^{1}(\Omega)\right) \times H_{0}^{1}(\Omega)$ (see [13], chapter 7). 
In a very similar way, one may obtain easily the so-called topological derivative (see [17]) associated to $J_{\lambda}$. The adjoint method introduced in [9] leads to the following result.

TheOREM 2.4 For any $\boldsymbol{x}_{\mathbf{0}} \in \Omega$ and $\rho \in \mathbb{R}^{+}$such that $D\left(\boldsymbol{x}_{\mathbf{0}}, \rho\right)=\left[\boldsymbol{x}_{\mathbf{0}}-\rho / 2, \boldsymbol{x}_{\mathbf{0}}+\right.$ $\rho / 2] \subset \Omega$, the functional $J_{\lambda}$ associated to $\mathcal{X}_{\Omega \backslash D\left(\boldsymbol{x}_{\mathbf{0}}, \rho\right)}$ may be expressed as follows :

$$
J_{\lambda}\left(\mathcal{X}_{\Omega \backslash D\left(\boldsymbol{x}_{\mathbf{0}}, \rho\right)}\right)=J_{\lambda}\left(\mathcal{X}_{\Omega}\right)+\rho\left(\frac{1}{2} \int_{0}^{T} v_{\Omega}^{2}\left(\boldsymbol{x}_{\mathbf{0}}, t\right) d t-\lambda\right)+o(\rho)
$$

in term only of the HUM control $v_{\Omega}$ associated to (1) with $\omega=\Omega$. The term factor of $\rho$ is called the topological derivative of $J_{\lambda}$ at the point $\boldsymbol{x}_{\mathbf{0}}$.

\section{Minimization of $J_{\lambda}$ in the level set framework}

Thanks to the previous computations, we are now in position to apply a gradient descent method for the minimization of the objective function $J_{\lambda}$ with respect to the position and shape of $\omega$. Precisely, from the relation (13), a descent direction is found by defining on $\partial \omega$, the vector field $\boldsymbol{\theta}$ as follows :

$$
\boldsymbol{\theta}=\left(\frac{1}{2} \int_{0}^{T} v_{\omega}^{2}(\boldsymbol{x}, t) d t-\lambda\right) \boldsymbol{\nu} \equiv j_{\lambda}\left(v_{\omega}\right) \boldsymbol{\nu} \quad \text { on } \quad \partial \omega
$$

and then we update the shape $\omega$ as $\omega^{\eta}=(I d+\eta \boldsymbol{\theta})(\omega)$. The parameter $\eta>0$ denotes a descent step small enough so that the following formal relation

$$
J_{\lambda}\left(\mathcal{X}_{\omega \eta}\right)=J_{\lambda}\left(\mathcal{X}_{\omega}\right)-\eta \int_{\partial \omega}\left(j_{\lambda}\left(v_{\omega}\right)^{2}\right) d \sigma+o(\eta)
$$

guarantees the decrease of $J_{\lambda}$. This method can be implemented in the Lagrangian framework: it suffices to mesh the domains $\omega, \Omega \backslash \omega$ and then to advect the mesh according to the descent direction $\boldsymbol{\theta}$ defined on $\partial \omega$ by (25). This imposes to mesh the moving interface $\partial \omega$. Furthermore, the change of topology of $\partial \omega$ is quite difficult to handle with this approach. In this respect, following recent works $([1,4,20])$, we adopt an Eulerian approach and we use a level-set method to capture the shape $\omega$ on a fixed mesh. Let us briefly recall the main aspect of this method. The level set approach (see $[18,19]$ for a survey) consists in giving a description of the evolving interface $\partial \omega$ which is independent of the discretizing mesh on $\Omega$. We define the level-set function $\psi$ in $\Omega$ such that

$$
\psi(\boldsymbol{x}) \leq 0 \quad \boldsymbol{x} \in \omega, \quad \psi(\boldsymbol{x})=0 \quad \boldsymbol{x} \in \partial \omega, \quad \psi(\boldsymbol{x}) \geq 0 \quad \boldsymbol{x} \in \Omega \backslash \omega .
$$

Therefore, the evolving interface $\partial \omega$, is characterized by

$$
\partial \omega=\{\boldsymbol{x}(\tau) \in \Omega \text { such that } \psi(\boldsymbol{x}(\tau), \tau)=0\},
$$

where $\tau$ designs a pseudo-time variable, increasing with time, that may be the real time, a load factor or in our case, the iterations of a given algorithm. Differentiation in $\tau$ of (28) then leads to

$$
\frac{\partial \psi}{\partial \tau}(\boldsymbol{x}(\tau), \tau)+\nabla \psi(\boldsymbol{x}(\tau), \tau) \cdot \frac{d \boldsymbol{x}(\tau)}{d \tau}=0 \quad \text { in } \quad \Omega
$$


Denoting by $\boldsymbol{F}$ the speed in the outward normal direction, such that $\frac{d \boldsymbol{x}(\tau)}{d \tau} \cdot \boldsymbol{\nu}=$ $\boldsymbol{F}(\boldsymbol{x}(\tau))$ where $\boldsymbol{\nu}=\nabla \psi /|\nabla \psi|$, we obtain the following nonlinear Hamilton-Jacobi equation of first order for $\psi$ :

$$
\frac{\partial \psi}{\partial \tau}(\boldsymbol{x}, \tau)+\boldsymbol{F}(\boldsymbol{x}, \tau)|\nabla \psi(\boldsymbol{x}, \tau)|=0, \quad \text { given } \quad \psi(\boldsymbol{x}, \tau=0) .
$$

Assuming that the shape $\partial \omega$ evolves in pseudo-time $\tau$ with the normal velocity $\boldsymbol{F}=$ $-j_{\lambda}\left(v_{\omega}\right) \boldsymbol{\nu}$ as proposed in (25), the transport of the level set function $\psi$ is therefore equivalent to moving the boundary of $\omega$ (the zero level-set of $\psi$ ) along the descent gradient direction $-\partial J_{\lambda} / \partial \omega$. Consequently, the partial differential system to solve is

$$
\left\{\begin{array}{l}
\frac{\partial \psi}{\partial \tau}-j_{\lambda}\left(v_{\omega}\right)|\nabla \psi|=0 \quad \text { in } \quad \Omega \times(0, \infty), \\
\psi(., \tau=0)=\psi_{0} \quad \text { in } \quad \Omega, \quad \psi=\psi_{0}>0 \quad \text { on } \quad \partial \Omega \times(0, \infty) .
\end{array}\right.
$$

We further impose that $\psi$ be constant and positive on $\Omega$ in order to ensure that $\partial \omega \cap \partial \Omega=\emptyset$. Finally, because of its advection, the level-set function may become too flat or too steep yielding either large errors in the location of its zero level or large errors in the evaluation of its gradient by finite differences. Therefore, a standard trick (see [4]) consists of replacing the level-set $\psi$ at the pseudo time $\tau_{0}$ by the regularized one, solution of the following problem

$$
\left\{\begin{array}{l}
\frac{\partial \tilde{\psi}}{\partial \tau}+\operatorname{sign}\left(\psi\left(\tau_{0}\right)\right)(|\nabla \tilde{\psi}|-1)=0 \quad \text { in } \quad \Omega \times(0, \infty), \\
\tilde{\psi}(., \tau=0)=\psi\left(\tau_{0}\right) \quad \text { in } \quad \Omega, \quad \tilde{\psi}=\psi\left(\tau_{0}\right) \quad \text { on } \quad \partial \Omega \times(0, \infty)
\end{array}\right.
$$

admitting as a stationary solution the signed distance to the initial interface $\left\{\psi\left(\boldsymbol{x}, \tau_{0}\right)=\right.$ $0\}$.

Remark 2 The behavior of the level set function is therefore strongly linked with the asymptotic behavior in the pseudo-time variable $\tau$ of the system (31). This question seems difficult and largely open. Some results in this direction exist under some restrictive assumptions on $\boldsymbol{F}$ (see [3] and the references therein).

Consequently, the descent algorithm to solve numerically the problem $\left(\mathcal{P}_{\omega}\right)$ in the framework of the level set approach may be structured as follows : $\Omega \in \mathbb{R},\left(y^{0}, y^{1}\right) \in$ $\left(H^{2}(\Omega) \cap H_{0}^{1}(\Omega)\right) \times H_{0}^{1}(\Omega), \varepsilon_{1}<<1$ and $T$ large enough be given,

(i) Meshing once for all of the fixed domain $\Omega$. Initialization of the level-set $\psi^{0}$ corresponding to an initial guess $\omega^{0} \in \Omega$ such that $\left|\omega^{0}\right|=L|\Omega|$;

(ii) For $k \geq 0$, iteration until convergence (i.e. $\left|J_{\lambda}\left(\mathcal{X}_{\omega^{k+1}}\right)-J_{\lambda}\left(\mathcal{X}_{\omega^{k}}\right)\right| \leq \varepsilon_{1} J_{\lambda}\left(\mathcal{X}_{\omega^{0}}\right)$ ) as follows:

- Computation of the HUM control $v_{\omega^{k}}$ associated to the domain $\omega^{k}$. The HUM control is obtained by solving the linear system (6) with a conjugate gradient algorithm (CG).

- Computation on $\Omega$ of the integrand $j_{\lambda}\left(v_{\omega^{k}}\right)$ according to the relation (13).

- Deformation of the shape by solving the transport Hamilton-Jacobi system (31). The new domain $\omega^{k+1}$ is characterized by the level-set function $\psi^{k+1}$ solution of (31) after a pseudo-time step $\Delta \tau^{k}$ starting from the initial function $\psi^{k}$ with velocity $-j_{\lambda}\left(v_{\omega^{k}}\right)$. The pseudo-time step $\Delta \tau^{k}$ is chosen according to the stability condition (40). 
(iii) From time to time, for stability reasons, re-initialization of the level-set function $\psi$ by solving (32).

At each iteration, the Lagrange multiplier $\lambda=\lambda^{k}$ is chosen so that the length of $\omega$ remains constant: $\left\|\mathcal{X}_{\omega^{k}}\right\|_{L^{1}(\Omega)}-\left\|\mathcal{X}_{\omega^{k+1}}\right\|_{L^{1}(\Omega)}=0, \forall k \geq 0$. From the relation $\omega^{k+1}=\left(I+\eta \boldsymbol{\theta}^{\boldsymbol{k}}\right) \omega^{k}$, we obtain

$$
\left\|\mathcal{X}_{\omega^{k+1}}\right\|_{L^{1}(\Omega)}-\left\|\mathcal{X}_{\omega^{k}}\right\|_{L^{1}(\Omega)}=\eta \int_{\omega^{k}} \operatorname{div}\left(\boldsymbol{\theta}^{k}\right) d x .
$$

We deduce from (25) that

$$
\lambda^{k}=\frac{1}{2} \int_{\omega^{k}} \operatorname{div}\left(\int_{0}^{T} v_{\omega^{k}}^{2}(\boldsymbol{x}, t) d t \boldsymbol{\nu}^{k}\right) d x / \int_{\omega^{k}} \operatorname{div}\left(\boldsymbol{\nu}^{k}\right) d x
$$

where the normal $\boldsymbol{\nu}^{k}$ is extended to the whole domain $\Omega$ as follows : $\boldsymbol{\nu}^{\boldsymbol{k}}=\nabla \psi^{k} /\left|\nabla \psi^{k}\right|$.

Furthermore, the topological derivative (24) permits to initialize efficiently the domain $\omega^{0}$. It suffices to compute the HUM control $v_{\Omega}$ corresponding to $\omega=\Omega$. This gives the distribution of the function $\boldsymbol{x}_{\mathbf{0}} \rightarrow 1 / 2 \int_{0}^{T} v_{\Omega}^{2}\left(\boldsymbol{x}_{\mathbf{0}}, t\right) d t$ in $\Omega$. Then, the domain $\omega^{0}$ is defined by

$$
\omega^{0}=\left\{\boldsymbol{x} \in \Omega, \frac{1}{2} \int_{0}^{T} v_{\Omega}^{2}(\boldsymbol{x}, t) d t-\lambda>0\right\}
$$

where $\lambda$ is chosen so that the length $\left\|\mathcal{X}_{\omega^{0}}\right\|_{L^{1}(\Omega)}$ of $\omega^{0}$ be equal to $L|\Omega|$. The level set function $\psi^{0}$, negative in $\omega^{0}$, is then $\psi^{0}(\boldsymbol{x})=\lambda-\frac{1}{2} \int_{0}^{T} v_{\Omega}^{2}(\boldsymbol{x}, t) d t$ for all $\boldsymbol{x}$ in $\Omega$.

\section{Numerical Approximation}

The main part of the algorithm is the computation of the HUM control associated to $\omega$. It is well-known that such a problem is extremely sensitive to the numerical approximation. We briefly describe in this section a recent, robust and efficient method to compute numerically this control (detailed in the appendix 8.1).

\subsection{Numerical approximation of the HUM control - Introduc- tion of additional vanishing terms}

Since the pioneering work of Glowinski-Li-Lions [10], the numerical approximation of the HUM control is known to be extremely sensitive with respect to the parameters of approximations (we refer to [21] for a review on this aspect). For simplicity, let us choose to approximate the wave system (1) by a semi-discrete finite difference scheme. Let us consider $N \in \mathbb{N}, h=1 /(N+1)$ and a uniform grid $\left(x_{i}\right)_{(i=0, \ldots, N+1)}$ of $\Omega$. Let us denote by $\boldsymbol{v}_{\boldsymbol{h}}$ the control vector of the finite dimensional system

$$
\begin{cases}\left(\boldsymbol{y}_{\boldsymbol{h}}\right)_{t t}-\boldsymbol{\Delta}_{\boldsymbol{h}} \boldsymbol{y}_{\boldsymbol{h}}=\boldsymbol{v}_{\boldsymbol{h}} \mathcal{X}_{\omega}, & \Omega \times(0, T), \\ \boldsymbol{y}_{\boldsymbol{h}}=0, & \partial \Omega \times(0, T), \\ \left(\boldsymbol{y}_{\boldsymbol{h}}(0),\left(\boldsymbol{y}_{\boldsymbol{h}}\right)_{t}(0)\right)=\left(\boldsymbol{y}_{\boldsymbol{h}}^{\mathbf{0}}, \boldsymbol{y}_{\boldsymbol{h}}^{\mathbf{1}}\right), & \Omega,\end{cases}
$$

such that $\boldsymbol{y}_{\boldsymbol{h}}(T)=\left(\boldsymbol{y}_{\boldsymbol{h}}\right)_{t}(T)=0$ on $\Omega . \quad \boldsymbol{\Delta}_{\boldsymbol{h}}$ designates a consistent finite difference approximation of the operator $\Delta$. Following [10], the control $\boldsymbol{v}_{\boldsymbol{h}}$ of minimal 
$l^{2}$-norm may be obtained by solving the linear system $\boldsymbol{\Lambda}_{\boldsymbol{h}}\left(\boldsymbol{\phi}_{\boldsymbol{h}}^{\mathbf{0}}, \boldsymbol{\phi}_{\boldsymbol{h}}^{\mathbf{1}}\right)=\left(\boldsymbol{y}_{\boldsymbol{h}}^{\mathbf{1}}, \boldsymbol{y}_{\mathbf{1}}^{\mathbf{0}}\right)$ - semi discrete version of (6)- using a conjugate gradient algorithm. $\boldsymbol{\Lambda}_{\boldsymbol{h}}$ designates the discrete HUM matrix. Let us note $P\left(\boldsymbol{v}_{\boldsymbol{h}}\right)$ the piecewise linear function on $\Omega$ such that $\left(P\left(\boldsymbol{v}_{\boldsymbol{h}}\right)\right)\left(x_{i}\right)=\left(v_{h}\right)_{i}, i=1, \ldots, N+$. Numerical simulations $([6,14])$ highlight that the behavior of $e_{h}=\left\|v-P\left(\boldsymbol{v}_{\boldsymbol{h}}\right)\right\|_{L^{2}(\omega \times(0, T))}$ with respect to the parameter of discretization $h$ crucially depends on the approximation $\boldsymbol{\Delta}_{\boldsymbol{h}}$ of $\Delta$. For instance, if the centered five point approximation is used for $\boldsymbol{\Delta}_{\boldsymbol{h}}$, then the error $e_{h}$ blows up exponentially with $1 / h$. This implies a wrong approximation of the descent direction $j_{\lambda}\left(v_{\omega}\right)$ and $a$ fortiori a divergence of the descent algorithm of the section 3 ! This non commuting property between exact controllability and numerical approximation is due, for the wave equation, to the spurious high frequency oscillations generated by the discrete dynamics (36). These spurious oscillations propagates with a so called group velocity of order $h$ and therefore can not be controlled in a uniform (with respect to $h$ ) time $t=T$. In practice, we observe that the conditioning number of $\boldsymbol{\Lambda}_{\boldsymbol{h}}$ behaves like $O\left(e^{1 / h}\right)$ and imply a divergence of the conjugate gradient algorithm (for $h$ small enough). In order to restore the uniform convergence of the discrete control (and therefore expect a convergence of the level function $\boldsymbol{\psi}_{\boldsymbol{h}}^{\boldsymbol{k}}$ - approximation of $\psi^{k}$ (see next section) - with respect to $k$ ), it is necessary to increase the group velocity of the high frequencies component. An efficient remedy consists in considering the approximation (using standard finite difference or finite element method) of the so-called viscous wave equation

$$
y_{t t}-\Delta y+\frac{h^{2}}{4} \Delta\left(y_{t t}\right)=v_{\omega} \mathcal{X}_{\omega}, \quad \Omega \times(0, T)
$$

consistent with (1) and which group velocity is uniformly (with respect to $h$ ) bounded by below for all the components. Using a Fourier analysis [14] or a semi-discrete multiplier technique [6], one may obtain the following result.

THEOREM 4.1 The semi-discrete scheme associated to (37) is uniformly controllable with respect to $h$. In addition, if $\left(P\left(\boldsymbol{y}_{\boldsymbol{h}}^{\mathbf{0}}\right), P\left(\boldsymbol{y}_{\boldsymbol{h}}^{\mathbf{1}}\right)\right)$ converge strongly toward $\left(y^{0}, y^{1}\right)$ in $H_{0}^{1}(\Omega) \times L^{2}(\Omega)$ as $h$ goes to 0 , then the corresponding control $\boldsymbol{v}_{\boldsymbol{h}}$ of minimal $l^{2}$-norm is such that $\lim _{h \rightarrow 0}\left\|P\left(\boldsymbol{v}_{\boldsymbol{h}}\right)-v\right\|_{L^{2}(\omega \times(0, T))}=0$.

The conditioning number cond $\left(\boldsymbol{\Lambda}_{\boldsymbol{h}}\right)$ of the discrete HUM matrix associated to this modified scheme is of order of $h^{-2}$. In practice, this modification leads to a very fast convergence of the conjugate gradient algorithm, obtained after few iterations independent of the value of $h$. Moreover, on the square domain where the eigenvalues and eigenvectors of the discrete scheme are known, the control $\boldsymbol{v}_{\boldsymbol{h}}$ may be solved exactly in time via a spectral method. This is detailed in the appendix 8.2. For general domain, a time discretization is necessary and we refer to [14] for uniformly controllable (with respect to both $h$ and $\Delta t$ ) full discrete approximation of (1).

\subsection{Resolution of the Hamilton-Jacobi equation}

Let us now briefly consider the resolution of the non-linear Hamilton-Jacobi equation (31). We introduce a parameter $\Delta \tau>0$ and note by $\boldsymbol{\psi}_{\boldsymbol{h}}^{\boldsymbol{k}}$ the approximation of the function $\psi$ at the point $x_{i}$ and at the pseudo-time $\tau=k \Delta \tau$. We note $\boldsymbol{j}_{\boldsymbol{\lambda} \boldsymbol{h}}$ the 
approximation of $j_{\lambda}\left(v_{\omega}^{k}\right)$ such that

$$
j_{\lambda \boldsymbol{h}}=\frac{1}{2} \int_{0}^{T}\left(\boldsymbol{v}_{\boldsymbol{h}}\right)^{2} d t-\lambda .
$$

The hyperbolic system (31) is then solved using an explicit weighted essentially nonoscillatory scheme of order one in pseudo-time $\tau$ and of order two in space (see [18, 19] for a complete description):

$$
\frac{\boldsymbol{\psi}_{\boldsymbol{h}}^{\boldsymbol{k}+\boldsymbol{1}}-\boldsymbol{\psi}_{\boldsymbol{h}}^{\boldsymbol{k}}}{\Delta \tau}+\left(\max \left(-\boldsymbol{j}_{\boldsymbol{\lambda} \boldsymbol{h}}\left(v_{\omega^{k}}\right), 0\right) \nabla_{k}^{+}+\min \left(-\boldsymbol{j}_{\boldsymbol{\lambda} \boldsymbol{h}}\left(v_{\omega^{k}}\right), 0\right) \nabla_{k}^{-}\right)=0, \quad k \geq 0
$$

where $\left(\nabla_{k}^{+}, \nabla_{k}^{-}\right)$designates forward and upward approximation of $\left|\nabla \psi^{k}\right|$. This explicit scheme is stable under the condition $\Delta \tau \leq h / \max _{\Omega}\left|\boldsymbol{j}_{\boldsymbol{\lambda} h}\left(v_{\omega^{k}}\right)\right|$. In order that the pseudo-time step $\Delta \tau$ decreases with respect to the iterations $k$, we consider the following pseudo-time step

$$
\Delta \tau^{k}=\min \left(1, \max _{\Omega}\left|\boldsymbol{j}_{\boldsymbol{\lambda} \boldsymbol{h}}\left(v_{\boldsymbol{\omega}^{k}}\right)\right|\right) \frac{h}{\max _{\Omega}\left|\boldsymbol{j}_{\boldsymbol{\lambda} \boldsymbol{h}}\left(v_{\boldsymbol{\omega}^{k}}\right)\right|} \quad\left(\leq \Delta \tau^{k-1}\right), \quad \forall k>0 .
$$

Remark 3 The upwind scheme (39) is motivated by the propagation of information through characteristics in the first order hyperbolic equation (30). Very interestingly with respect to the discussion of the previous section, this scheme may be replaced by usual centered finite differences ones, provided the addition of an artificial viscosity term (see [18]) (namely the approximation of $\psi_{\tau}+F|\nabla \psi|=h \Delta \psi$ instead of (30)). The reason to introduce this term here is however different.

\section{$5 \quad$ Numerical experiments}

In this section, we present some numerical experiments in order to evaluate the efficiency of the previous algorithm to find local minima of the functional $J_{\lambda}$. Since the computation of the HUM control (via the resolution of (6)) is more expensive in CPU time than the resolution of the Hamilton-Jacobi (31) system, we perform two explicit pseudo-time steps of (31) after each resolution of (6). During these steps, we perform two re-initialization of the level set function by solving (32). Moreover, in the algorithm, we use $\varepsilon_{1}=10^{-6}$ and values of $h$ smaller than $1 / 300$.

\subsection{Example 1: Observability constant}

Let us consider the simplest initial condition on $\bar{\Omega}=[0,1]$

$$
y^{0}(\boldsymbol{x})=\sin (\pi \boldsymbol{x}) ; \quad y^{1}(\boldsymbol{x})=0 .
$$

Some calculations permits to obtain that the HUM control $v_{\Omega}$ acting on the whole domain is given by

$$
v_{\Omega}(\boldsymbol{x}, t)=\frac{-\pi^{2}}{\pi^{2} T^{2}-\sin (\pi T)^{2}}\left(\sin (\pi T)^{2} \cos (\pi t)-(\cos (\pi T) \sin (\pi T)+\pi T) \sin (\pi t)\right) y^{0}(\boldsymbol{x})
$$



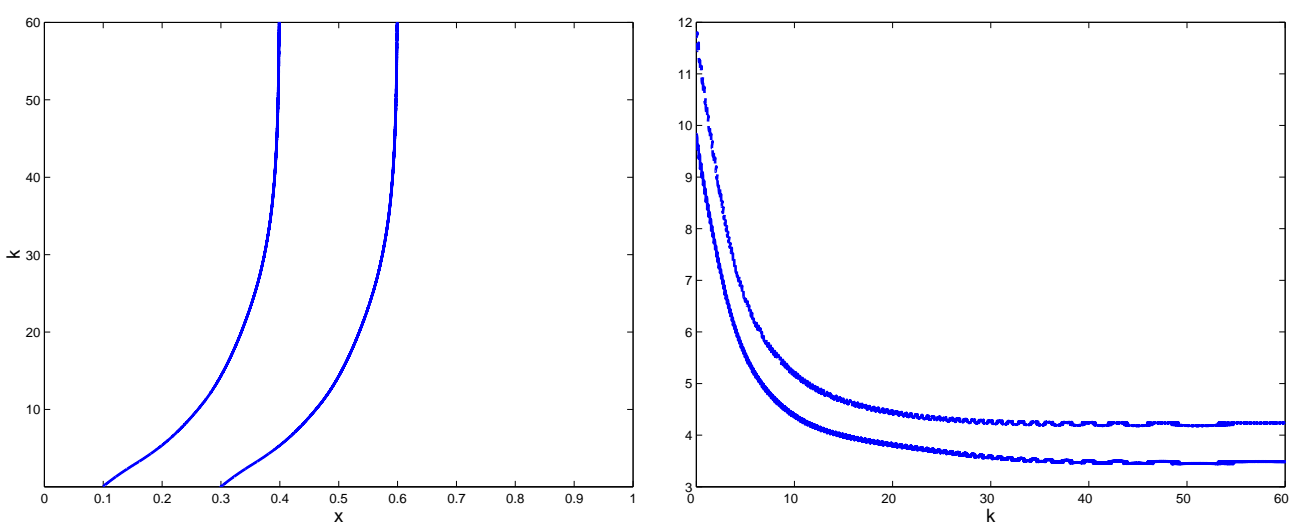

Figure 1: $T=3-L=0.2$. Evolution of the Figure 2: Evolution of the $\operatorname{cost} J\left(\mathcal{X}_{\omega^{k}}\right)$ (top) zeros of $\psi^{k}$ vs $k$. and of the ratio (44) (bottom) vs. $k$.

leading to

$$
\frac{1}{2} \int_{0}^{T} v_{\Omega}^{2}(\boldsymbol{x}, t) d t=\pi^{3} \frac{\pi T+\cos (\pi T) \sin (\pi T)}{\pi^{2} T^{2}-\sin (\pi T)^{2}}\left(y^{0}(\boldsymbol{x})\right)^{2} ;
$$

Consequently, it follows from the relation (24) that the optimal position for $\omega$ with an arbitrarily small length is the centered interval on $\Omega$ (provided that $T>T^{\star}(\Omega \backslash \omega)$ ). This is what we obtain numerically, for any initialization $\psi^{0}$ of the level set function. This result still holds for any value of $|\omega|<1$ (non necessary small). For this particular choice of initial condition $\left(y^{0}, y^{1}\right)$, the functional to minimize is strictly convex: the minimum is unique and the original problem $\left(\mathcal{P}_{\omega}\right)$ is well-posed. We take $L=0.2$ and $T=3$. Figure 1 depicts the evolution of the zeros of the level set function $\psi^{k}$ in function of $k$, arbitrarily initialized by $\psi^{0}(\boldsymbol{x})=(\boldsymbol{x}-0.1)(\boldsymbol{x}-0.3)$. The limit domain is $\omega^{\text {lim }}=[1 / 2-L / 2,1 / 2+L / 2]=[0.4,0.6]$. Figure 2 depicts the corresponding evolution of $J\left(\omega^{k}\right)$ and of the ratio

$$
\frac{\left\|\phi_{\omega^{k}}^{0}\right\|_{L^{2}(\Omega)}^{2}+\left\|\phi_{\omega^{k}}^{1}\right\|_{H^{-1}(\Omega)}^{2}}{\int_{\omega^{k}} \int_{0}^{T}\left(\phi_{\omega^{k}}(\boldsymbol{x}, t)\right)^{2} d t d x}
$$

which appears in (5). The fact that this ratio decreases implies that the domain $\omega^{k+1}$ leads to a better estimation of the norm $\left\|\left(\phi^{0}, \phi^{1}\right)\right\|_{L^{2} \times H^{-1}}$ than $\omega^{k}$. Thus and as expected, by minimizing the $L^{2}$-norm of the exact control with respect to its support, we increase the observability/controllability character of the wave system (we remind that the constant $C_{T}$ in (5) is arbitrarily large if (1) is not exactly controllable with the couple $(\omega, T))$. Practically, this implies that if (1) is controllable for $\left(\omega^{k}, T\right)$ at the iteration $k$, then (1) remains exactly controllable for $\left(\omega^{k+1}, T\right)=\left(\left(I+\eta \boldsymbol{\theta}^{\boldsymbol{k}}\right)\left(\omega^{k}\right), T\right)$ where $\boldsymbol{\theta}^{k}$ is such that $J\left(\mathcal{X}_{\omega^{k+1}}\right) \leq J\left(\mathcal{X}_{\omega^{k}}\right)$. The descent algorithm conserves the controllability property.

We check on this example the decrease of $J$ with respect to the inclusion of $\omega$ and with respect to $T$. Figure 3 depicts the variation of $\log \left(J\left(\mathcal{X}_{\omega}, T\right)\right)$ with respect to $\log (T)$ for $\omega=[0.4,0.6]$. For $T$ large enough, we obtain $J\left(\mathcal{X}_{\omega}, T\right) \approx O\left(T^{-1.007}\right)$ in agreement with (43). Figure 4 depicts the variation of $\log \left(J\left(\mathcal{X}_{\omega_{\rho}}\right)\right)$ with respect to 

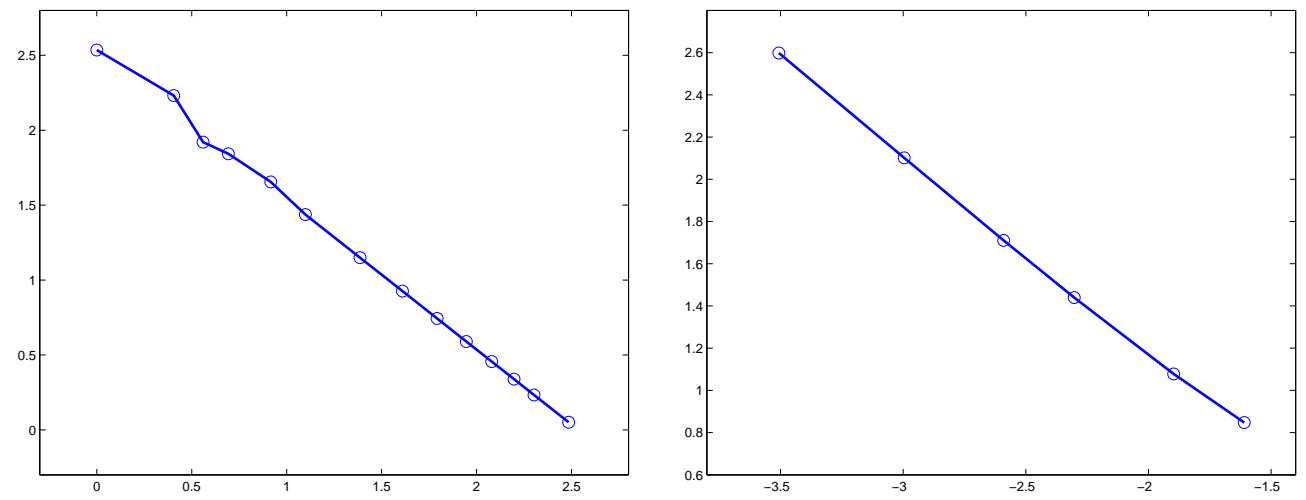

Figure 3: $\omega=[0.4,0.6]-\log \left(J\left(\mathcal{X}_{\omega}, T\right)\right)$ vs Figure 4: $T=3-\omega_{\rho}=[1 / 2-\rho, 1 / 2+\rho]$ $\log (T)-T \in[1,12]$.

$\log \left(J\left(\mathcal{X}_{\omega_{\rho}}\right)\right)$ vs $\log (\rho)-\rho \in[0.03,0.25]$.

$\rho$ for $\omega_{\rho}=[1 / 2-\rho, 1 / 2+\rho]$ and $T=3$. For $\rho$ small enough, we obtain $J\left(\mathcal{X}_{\omega_{\rho}}\right)=$ $O\left(\rho^{-0.997}\right)$ (we may also check numerically the relation (24)).

\subsection{Example 2: Dependence with respect to the time $T$}

Still with $\Omega=(0,1)$, we consider the following regular initial condition, on $\bar{\Omega}$

$$
y^{0}(\boldsymbol{x})=e^{-100(\boldsymbol{x}-0.3)^{2}} \mathcal{X}_{\Omega}(\boldsymbol{x}) ; \quad y^{1}(\boldsymbol{x})=0 .
$$

Moreover, we take $L=0.16$. Let us first use the relation (24). The function $1 / 2 \int_{0}^{T} v_{\Omega}^{2}(\boldsymbol{x}, t) d t$ is depicted on figure 5 -left for $T=1.5,2.5$ and $T=3$. For each values of $T$, the functions presents local minima highlighting that the optimal domain is very likely composed of several disjoint sub-intervals. For instance for $T=2.5$, the prediction $\omega^{0}$ of length $L$ defined from the relation (35) is composed of three sub-intervals : $[0.056,0.132] \cup[0.269,0.328] \cup[0.686,0.711]$. The corresponding value of the multiplier is $\lambda \approx 6.733$. We obtain $J\left(\mathcal{X}_{\omega^{0}}\right) \approx 22.491$. Figure 5 -right depicts the corresponding function $\psi_{o p t}^{0}(\boldsymbol{x})=-1 / 2 \int_{0}^{T} v_{\Omega}^{2}(\boldsymbol{x}, t) d t+\lambda$ we may choose to initialize the level set function. The prediction depends also on the time $T$. For $T=3$, we obtain a symmetric prediction $\omega^{0} \approx[0.071,0.107] \cup[0.278,0.322] \cup[0.678,0.722] \cup$ $[0.893,0.929]$ for which $J\left(\mathcal{X}_{\omega^{0}}\right) \approx 18.307(\lambda \approx 5.696)$. For $T=1.5$, the prediction is composed of two disjoints components $\omega^{0} \approx[0.668,0.737] \cup[0.858,0.949]$ for which $J\left(\mathcal{X}_{\omega^{0}}\right) \approx 33.638(\lambda \approx 11.26)$.

We now compare the result of the level set algorithm initialized either by $\psi_{o p t}^{0}$ or by functions of the type $\psi_{p}^{0}(\boldsymbol{x})=C_{p}-\sin ^{2}(p \pi \boldsymbol{x}), p \in \mathbb{N}$ and $C_{p}>0$ such that $\left\|\mathcal{X}_{\left(\psi_{p}^{0} \leq 0\right)}\right\|_{L^{1}(\Omega)}=L|\Omega|$. The corresponding $\omega_{p}^{0}$ is composed of $p$ disjoints components uniformly distributed on $\Omega$. Figure 6 depicts the zeros of $\psi_{p}^{k}$, for $k=1, \ldots, 100$ and the value $p=6$. The convergent domain $\omega_{p}^{\text {lim }}$, obtained after less than 100 iterations, is composed of three disjoints intervals and is in agreement with the prediction obtained from the relation (24) highlighting the interest of the topological derivative. The algorithm permits a significant reduction of the norm of the control, from $J\left(\mathcal{X}_{\omega^{0}}\right) \approx$ 28.11 to $J\left(\mathcal{X}_{\omega^{\text {lim }}}\right) \approx 22.65$. Figure 7 depicts the level set functions $\psi_{p}^{0}$ and $\psi_{p}^{100}$ and 

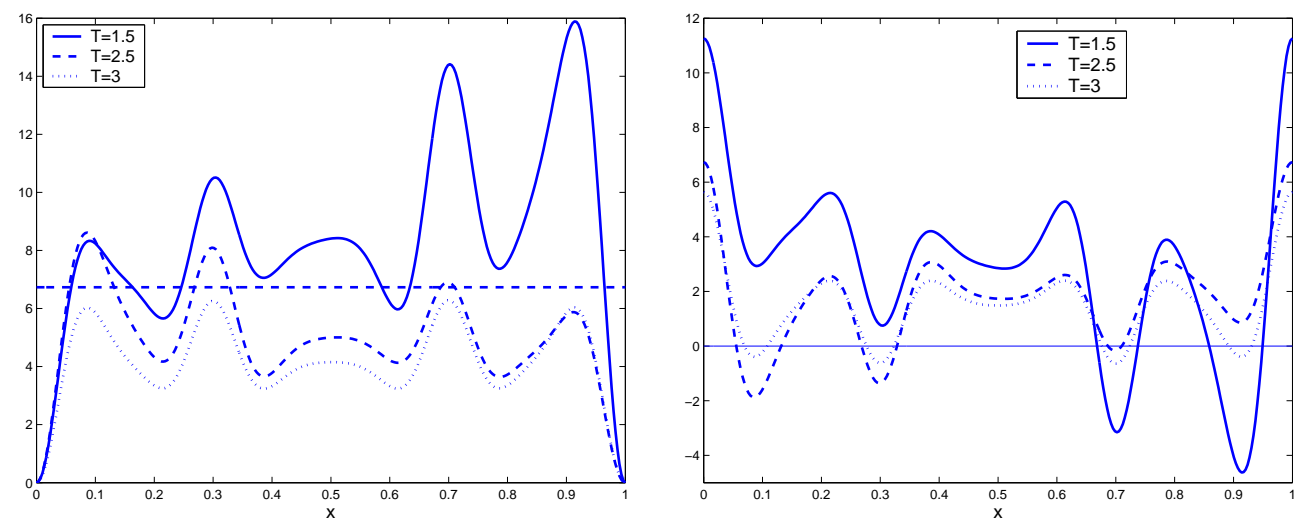

Figure 5: Left: Function $1 / 2 \int_{0}^{T} v_{\Omega}^{2}(\boldsymbol{x}, t) d t$ for $T=1.5,2.5$ and 3 - Right: Corresponding initial level set $\psi^{0}(\boldsymbol{x})=-1 / 2 \int_{0}^{T} v_{\Omega}^{2}(\boldsymbol{x}, t) d t+\lambda$.
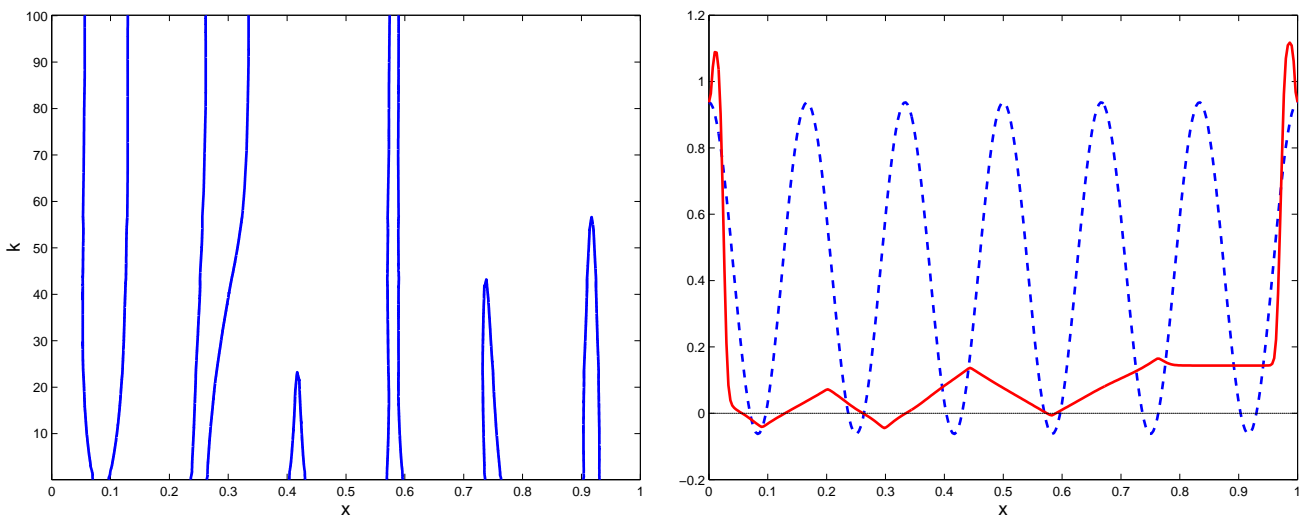

Figure 6: $p=6$ - Evolution of the zeros Figure 7: $p=6-T=2.5$ - Initial $\psi_{p}^{0}(--)$ of $\psi_{p}^{k}, k=1, \ldots, 100-J\left(\mathcal{X}_{\omega_{p}^{0}}\right) \approx 28.11$, and "limit" $\psi_{p}^{\text {lim }}(-)$ level set functions. $J\left(\mathcal{X}_{\omega_{p}^{100}}\right) \approx 22.65$. 

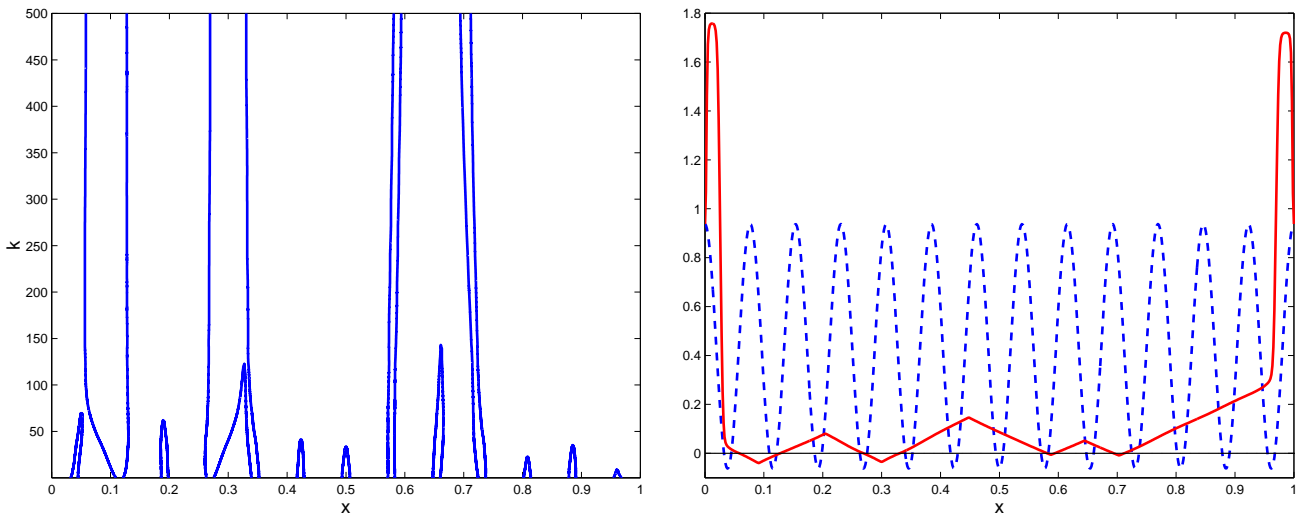

Figure 8: $p=13$ - Evolution of the zeros Figure 9: $p=13-T=2.5$ - Initial $\psi^{0}(--)$ of $\psi_{p}^{k}, k=1, \ldots, 500-J\left(\mathcal{X}_{\omega_{p}^{0}}\right) \approx 28.27$, and "limit" $\psi_{p}^{500}(-)$ level set functions. $J\left(\mathcal{X}_{\omega_{p}^{500}}\right) \approx 22.32$.

points out the effect of the regularization process (32) on the shape of $\psi_{p}^{100}$. Figures 8-9 depict the results obtained with $p=13$. This value provides a different local minimum $\omega$ and improves slightly the value of $J\left(\mathcal{X}_{\omega^{l i m}}\right) \approx 22.32$. This also put in evidence the dependence of the limit with respect to the initialization and the existence of several local minima. Figures 10-left represents the evolution of the energy of the wave system (1) and the $L^{2}(\omega)$-norm of the control with respect to time, associated to the initial domain $\omega_{p}^{0}$ (dashed curve) and to the limit one $\omega_{p}^{\text {lim }}$ (continuous curve). The uniform distributed initial domain $\omega_{p}^{0}$ produces a regular decrease of the energy. On the contrary, the control associated to the optimal configuration acts more on the second part of the time interval $[0, T]$.

Additional simulations with the values $T=1.5$ and $T=3$ produce similar results in agreement with the prediction $\omega_{p}^{0}$ given by the topological derivative. For $T=3$, Figure 11 and Figure 12 depicts the evolution of the zeros of $\psi_{p}^{k}$ initialized using the value $p=2$ and $p=13$ respectively. The second value provides the optimal global domain (composed of four intervals) while the first one leads to a local minimum (composed of two intervals).

Furthermore, for small values of $T$, a direct use of the relation (24) may be inappropriate to initialize efficiently the support of the control. We recall that this relation is valid for a couple $(\omega, T)$ for which system (1) is exactly controllable and may be applied if the length $|\omega|$ is small. These two conditions are incompatible if $T$ is small enough. Thus, for $T=0.5,(24)$ leads to an initial prediction $\omega^{0}$ concentrated around the point $\boldsymbol{x}=1 / 10$ (see figure 13). On the other hand, Figure 14 depicts the evolution of the level set function initialized using $p=13$ (a uniformly distribution of $p=13$ sub-intervals on $\Omega$ for which (1) is exactly controllable). The convergent domain $\omega_{p}^{\text {lim }}$, obtained after 150 iterations is composed of 7 sub-intervals distributed on the whole interval $\Omega$ so that $(1)$ be exactly controllable for the couple $\left(\omega_{p}^{\text {lim }}, T\right)$ (we check that the point $\boldsymbol{x}=1 / 10$ belongs to $\omega^{\text {lim }}$ ).

More generally, remark that the method we present permits to find numerically the exact HUM control of (1) associated to an arbitrarily small value of $T$ distributed on 

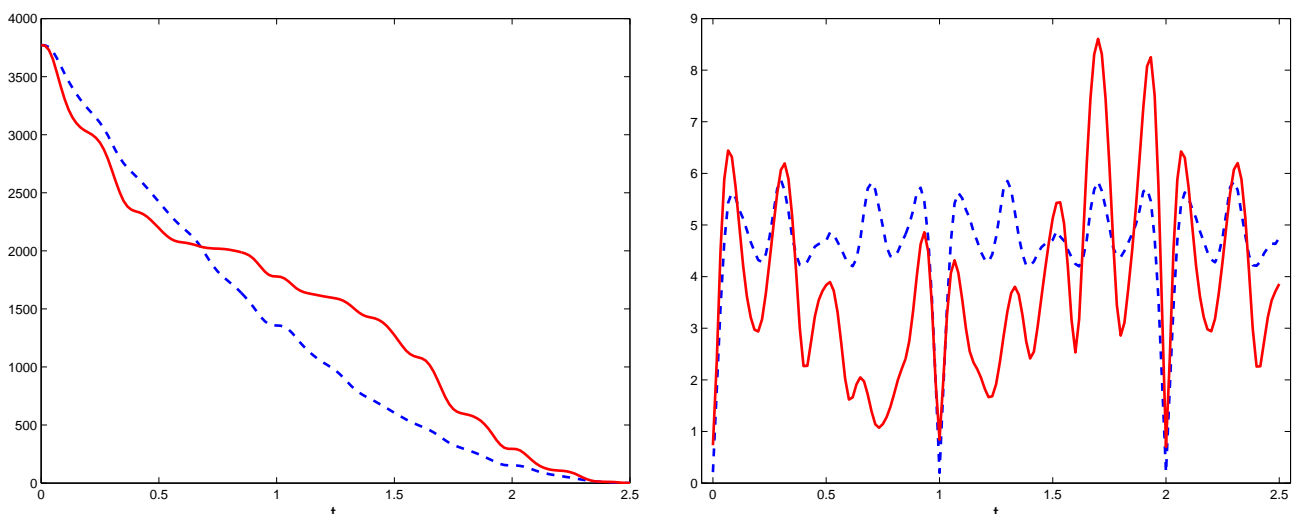

Figure 10: $p=13-T=2.5$ - Energy of the system (left) and $\left\|v_{\omega}\right\|_{L^{2}(\omega)}$ (right) vs. $t$ $E(T) / E(0) \approx 8.38 \times 10^{-7}$ corresponding to the initial level set function $\psi^{0}(--)$ and to the limit one $(-)$.
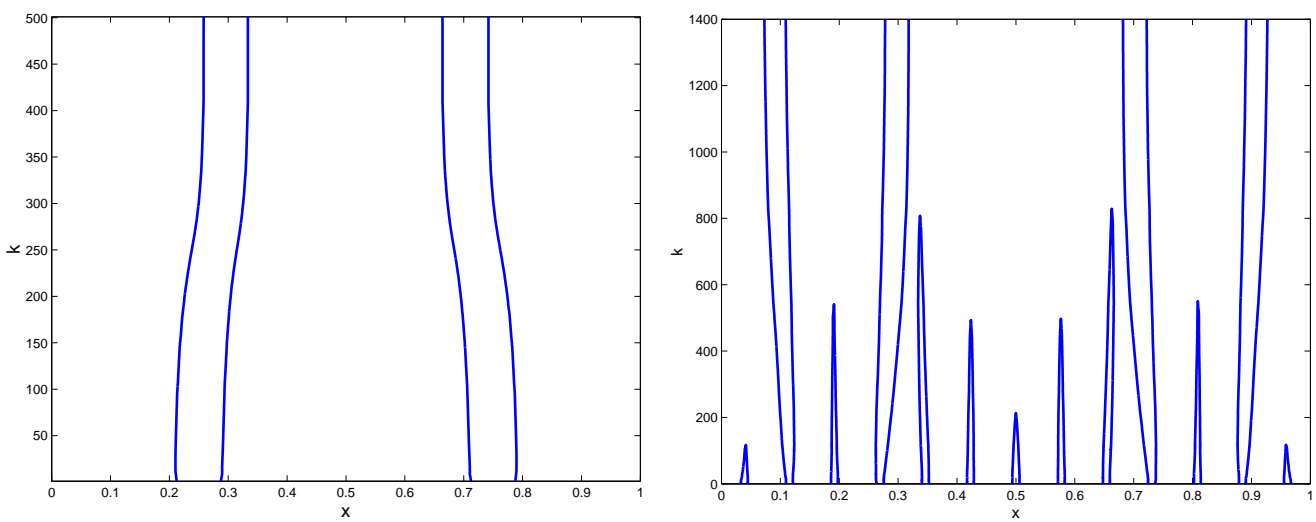

Figure 11: $T=3$ and $p=2$ - Evolution of Figure 12: $T=3$ and $p=13$ - Evolution of the zeros of $\psi_{p}^{k}$ for $k=1, \ldots, 500-J\left(\mathcal{X}_{\omega_{p}^{0}}\right) \approx$ the zeros of $\psi_{p}^{k}$ for $k=1, \ldots, 1400-J\left(\mathcal{X}_{\omega_{p}^{0}}\right) \approx$ $28.521, J\left(\omega_{p}^{500}\right) \approx 23.120$ $25.085, J\left(\mathcal{X}_{\omega_{p}^{1400}}\right) \approx 19.772$ 
$\omega_{T}$ (dependent on $T$ ) of arbitrarily small Lebesgue measure $\left|\omega_{T}\right|$ ! One may conjecture that the number of disjoints components of the optimal domain $\omega_{T}$ is an increasing function of $T^{-1}$.
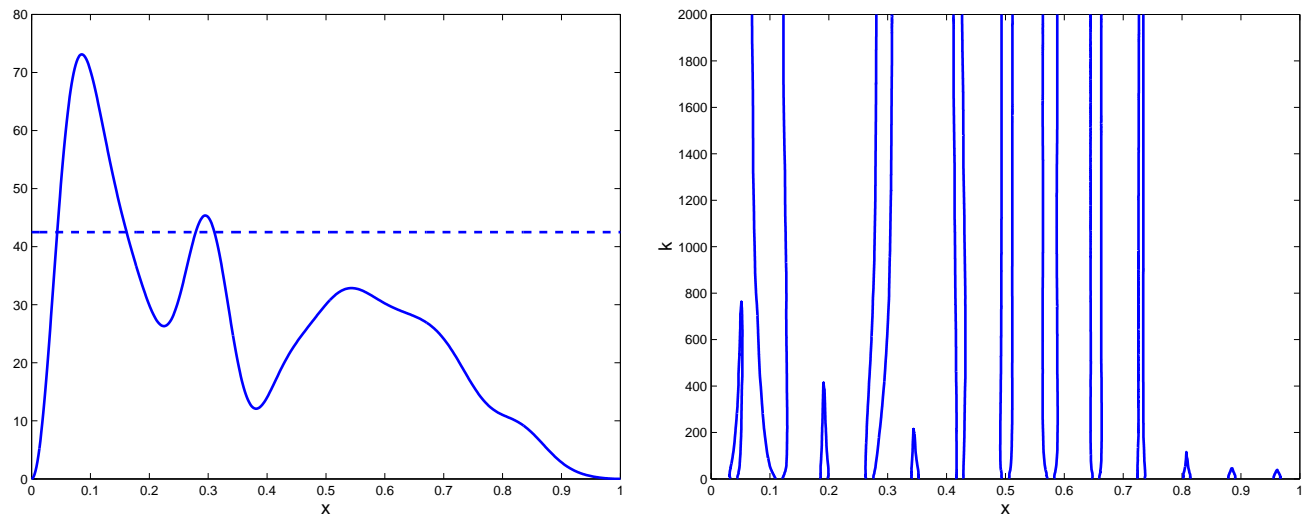

Figure 13: $T=0.5-1 / 2 \int_{0}^{T} v_{\Omega}^{2}(\boldsymbol{x}, t) d t$ in $\Omega$. Figure 14: $T=0.5-p=13$ - Evolution of the zeros of $\psi_{p}^{k}$ vs. $k$.

\section{Convexification of the problem $\left(\mathcal{P}_{\omega}\right)$}

We investigate in this section whether or not the problem $\left(\mathcal{P}_{\omega}\right)$ may be not wellposed: is there exist an initial condition $\left(y^{0}, y^{1}\right) \in H_{0}^{1}(\Omega) \times L^{2}(\Omega)$ for which the infimum cost function is not reached in the set of characteristic function. In such a case, the optimal sub-domain $\omega$ is composed of an arbitrarily large number of disjoint components and the original problem is thus non well-posed. The numerical detection of this phenomenon with the level set approach may be done as follows: (i) consider an initial level set function $\psi_{p}$ of the type $\left.\psi_{p}(\boldsymbol{x})=C_{p}-\sin ^{2}(p \pi \boldsymbol{x}), C_{p} \in\right] 0,1[$ associated to a domain $\omega_{p}$ composed of $p \in \mathbb{N}$ disjoint components uniformly distributed in $\Omega$; (ii) compute with the algorithm described above the limit level set function $\psi_{p}^{\text {lim }}$ associated to a domain $\omega_{p}^{\text {lim }}$; (iii) count the number $\sharp \omega_{p}^{\text {lim }}$ of disjoint components of $\omega_{p}^{l i m}$. Then, the variation of the function $p \rightarrow \sharp \omega_{p}^{\text {lim }}$ permits to detect the possible non well-posedness character of $\left(\mathcal{P}_{\omega}\right)$. However, for large values of $p$, this method requires very small values of $h$ and cannot be used. Another method consists in replacing the set $L^{\infty}(\Omega,\{0,1\})$ of characteristics function by it weak * closure $L^{\infty}(\Omega,[0,1])$ (or equivalently its convex envelop). We then define the convexified problem $\left(\mathcal{C P}_{\omega}\right)$ of $\left(\mathcal{P}_{\omega}\right)$ :

$$
\left(\mathcal{C} \mathcal{P}_{\omega}\right): \inf _{s \in L^{\infty}(\Omega ;[0,1])} \bar{J}_{\lambda}(s) \quad \text { with } \quad \bar{J}_{\lambda}(s)=\frac{1}{2} \int_{\Omega} s(\boldsymbol{x}) \int_{0}^{T} v_{s}^{2}(\boldsymbol{x}, t) d t d x+\lambda \int_{\Omega} s(\boldsymbol{x}) d x
$$


where $v_{s}$ (function of the density $s$ ) is such that $s v_{s}$ is the HUM control of minimal $L^{2}(\Omega \times(0, T))$ norm associated to

$$
\begin{cases}y_{t t}-\Delta y=s(\boldsymbol{x}) v_{s}, & \Omega \times(0, T) \\ y=0, & \partial \Omega \times(0, T) \\ \left(y(\cdot, 0), y_{t}(\cdot, 0)\right)=\left(y^{0}, y^{1}\right), & \Omega .\end{cases}
$$

Once again, the multiplier $\lambda$ is chosen so that $\int_{\Omega} s(\boldsymbol{x}) d x=L|\Omega|$, relaxed version of the condition $|\omega|=L|\Omega|$. Thus, the optimal shape design problem $\left(\mathcal{P}_{\omega}\right)$ is replaced by a simpler functional optimal problem. It is easy to see that problem $(\mathcal{C P} \omega)$ is well posed and admits solutions in $L^{\infty}(\Omega ;[0,1])$. Moreover, problems $\left(\mathcal{C P}_{\omega}\right)$ and $\left(\mathcal{P}_{\omega}\right)$ both coincide if and only if the optimal density $s$ for $\left(\mathcal{C P}_{\omega}\right)$ is a characteristic function. On the contrary, if the optimal density $s$ takes value in $] 0,1[$, this indicates that the original problem is not well-posed.

Similarly to problem $\left(\mathcal{P}_{\omega}\right)$, local minimal density may be obtained using a gradient descent method. For any $\eta \in \mathbb{R}^{+}$and any $s_{1} \in L^{\infty}(\Omega)$, we associate to the perturbation $s^{\eta}=s+\eta s_{1}$ the derivative of $\bar{J}_{\lambda}$ with respect to $s$ in the direction $s_{1}$ as follows:

$$
\frac{\partial \bar{J}_{\lambda}(s)}{\partial s} \cdot s_{1}=\lim _{\eta \rightarrow 0} \frac{\bar{J}_{\lambda}\left(s+\eta s_{1}\right)-\bar{J}_{\lambda}(s)}{\eta}
$$

and we obtain that :

Theorem 6.1 If $\left(y^{0}, y^{1}\right) \in\left(H^{2}(\Omega) \cap H_{0}^{1}(\Omega)\right) \times H_{0}^{1}(\Omega)$, then the derivative of $\bar{J}_{\lambda}$ with respect to $s$ in the direction $s_{1}$ exists and take the expression

$$
\frac{\partial \bar{J}_{\lambda}(s)}{\partial s} \cdot s_{1}=\int_{\Omega} s_{1}(\boldsymbol{x})\left(-\frac{1}{2} \int_{0}^{T} v_{s}^{2}(\boldsymbol{x}, t) d t-\lambda\right) d x
$$

This permits to define the following descent direction :

$$
s_{1}(\boldsymbol{x})=\frac{1}{2} \int_{0}^{T} v_{s}^{2}(\boldsymbol{x}, t) d x-\lambda, \quad \forall \boldsymbol{x} \in \Omega .
$$

Consequently, for any function $\eta \in L^{\infty}\left(\Omega, \mathbb{R}^{+}\right)$with $\|\eta\|_{L^{\infty}}$ small enough, we have $\bar{J}_{\lambda}\left(s+\eta s_{1}\right) \leq \bar{J}_{\lambda}(s)$. The multiplier $\lambda$ is determined so that, for any function $\eta \in$ $L^{\infty}\left(\Omega, \mathbb{R}^{+}\right)$and $\eta \neq 0,\left\|s+\eta s_{1}\right\|_{L^{1}(\Omega)}=L|\Omega|$ leading to

$$
\lambda=\frac{\left(\int_{\Omega} s(\boldsymbol{x}) d x-L|\Omega|\right)-\int_{\Omega} \eta(\boldsymbol{x}) \int_{0}^{T} v_{s}^{2}(\boldsymbol{x}, t) d t d x}{\int_{\Omega} \eta(\boldsymbol{x}) d x} .
$$

At last, the function $\eta$ is chosen so that $s(\boldsymbol{x})+\eta s_{1}(\boldsymbol{x}) \in[0,1]$ for all $\boldsymbol{x} \in \Omega$. A simple and efficient choice consists in taking $\eta=\varepsilon s(\boldsymbol{x})(1-s(\boldsymbol{x}))$ for all $\boldsymbol{x} \in \Omega$ with $\varepsilon$ a small real positive. Consequently, the descent algorithm to solve the convexified problem $\left(\mathcal{C} \mathcal{P}_{\omega}\right)$ may be structured as follows : let $\Omega \in \mathbb{R},\left(y^{0}, y^{1}\right) \in\left(H^{2}(\Omega) \cap H_{0}^{1}(\Omega)\right) \times H_{0}^{1}(\Omega)$, $L \in(0,1), T>T(\Omega)$ and $\varepsilon<1, \varepsilon_{1}<<1$ be given:

- Initialization of the density function $s^{0} \in L^{\infty}(\Omega ;] 0,1[)$; 
- For $k \geq 0$, iteration until convergence (i.e. $\left.\left|\bar{J}_{0}\left(s^{k+1}\right)-\bar{J}_{0}\left(s^{k}\right)\right| \leq \varepsilon_{1}\left|\bar{J}_{0}\left(s^{0}\right)\right|\right)$ as follows :

- Computation of the HUM control $s^{k} v_{s^{k}}$ corresponding to $s=s^{k}$.

- Computation of the descent direction $s_{1}^{k}$ defined by (50) where the multiplier $\lambda^{k}$ is defined by (51) with $\eta=\varepsilon s(1-s)$.

- Update of the density function in $\Omega$ :

$$
s^{k+1}=s^{k}+\varepsilon s^{k}\left(1-s^{k}\right) s_{1}^{k}
$$

with $\varepsilon \in \mathbb{R}^{+}$small enough in order to ensure the decrease of the cost function and $s^{k+1} \in L^{\infty}(\Omega,[0,1])$.

We now complete the section 5 with additional simulations using this new approach. Once again, we consider the simple initial condition (45) and choose to initialize the density $s$ by $s^{0}(\boldsymbol{x})=L=0.16$ in $\Omega$ (so that $\int_{\Omega} s^{0}(\boldsymbol{x}) d x=L|\Omega|$ ). We take $\varepsilon=10^{-2}$ and $\varepsilon_{1}=10^{-6}$.

Figure 15 depicts the evolution of the cost function $\bar{J}_{0}\left(s^{k}\right)$ with respect to $k$. Figure 16 depicts the evolution of the ratio

$$
\frac{\left\|\phi_{s^{k}}^{0}\right\|_{L^{2}(\Omega)}^{2}+\left\|\phi_{s^{k}}^{1}\right\|_{H^{-1}(\Omega)}^{2}}{\int_{\Omega} s^{k}(\boldsymbol{x}) \int_{0}^{T}\left(\phi_{s^{k}}(\boldsymbol{x}, t)\right)^{2} d t d x}
$$

in function of $k$, relaxed version of the ratio (44). Once again, this ratio is decreasing with respect to $k$.

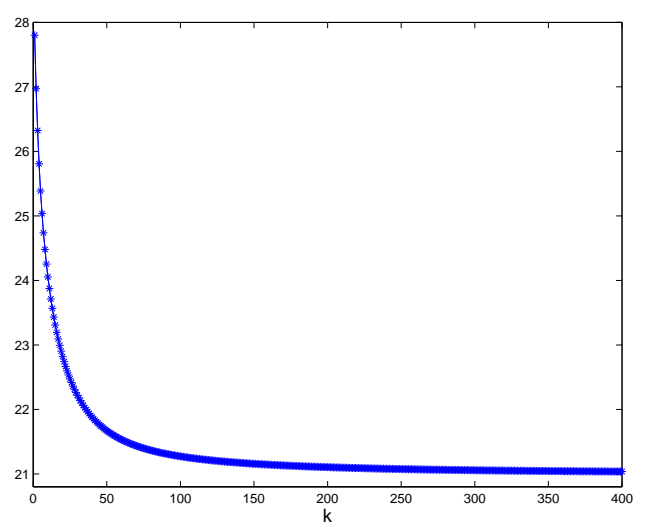

Figure 15: $T=3-\bar{J}_{0}\left(s^{k}\right)$ vs. $k$.

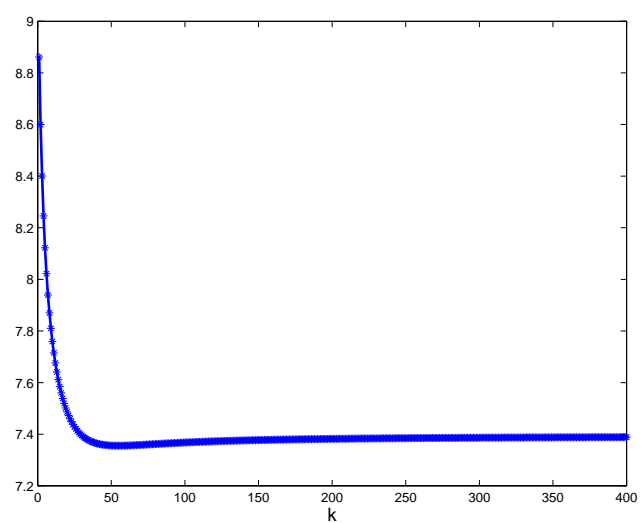

Figure 16: Corresponding ratio (53) vs $k$.

Figures 17 depicts the limit of the density $s^{k}$ for the cases $T=0.5,1.5,2.5$ and $T=$ 3. First, we observe that these limit densities are characteristic functions. Therefore, at least, for these data, the problem $\left(\mathcal{C P}_{\omega}\right)$ coincides with $\left(\mathcal{P}_{\omega}\right)$ which is well-posed. Secondly, we obtain the same limit domain than with the level-set approach (see figure 8 for $T=2.5$ and figure 12 for $T=3$ ).

We have not been able to exhibit an initial condition $\left(y^{0}, y^{1}\right) \in\left(H^{2}(\Omega) \cap H_{0}^{1}(\Omega)\right) \times$ $H_{0}^{1}(\Omega)$ (nor in $H_{0}^{1}(\Omega) \times L^{2}(\Omega)$ ) leading to a limit density $s^{\text {lim }}$ such that $\mid\{\boldsymbol{x} \in \Omega, 0<$ 

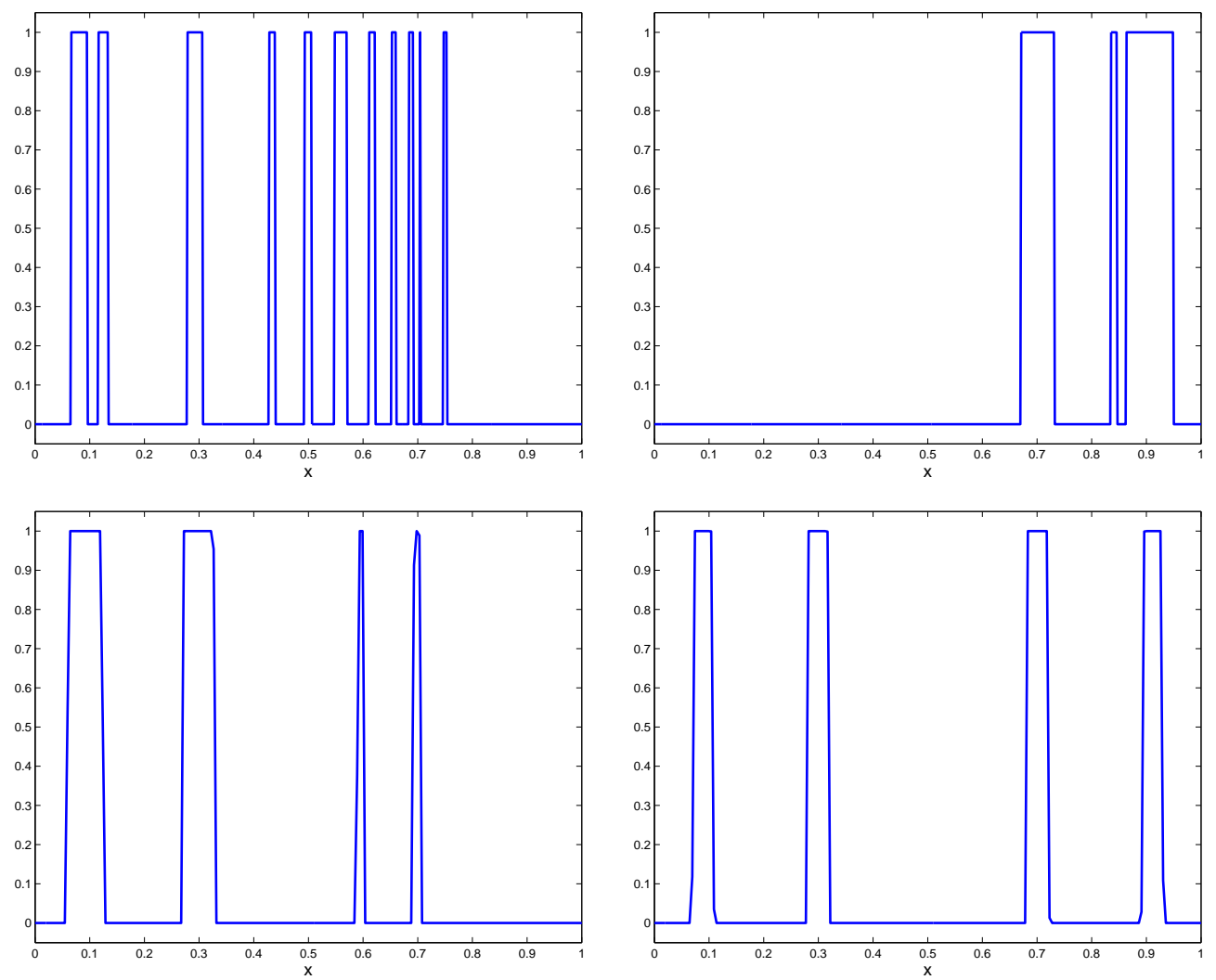

Figure 17: Limit density function $s^{\text {lim }}$ for $T=0.5$ (top left), $T=1.5$ (top right), $T=2.5$ (bottom left) and $T=3$ (bottom right) initialized with $s^{0}=L$ on $\Omega=(0,1)$ 
$s(\boldsymbol{x})<1\} \mid>0$. Therefore, we conjecture that the problem $\left(\mathcal{P}_{\omega}\right)$ is always wellposed: the infimum of $J$ is reached for a domain composed of a finite number of disjoints components. The introduction of the relaxed problem $\left(\mathcal{C P}_{\omega}\right)$ is however not useless: on the practical viewpoint, the algorithm described in this section appears more simple and less sensitive to numerical approximation than the algorithm based on the level set methodology. It avoids the resolution of Hamilton-Jacobi equation and provides a more regular decrease of the cost function.

\section{Concluding remarks}

We have presented a numerical method to obtain the optimal position of the distributed control for the 1-D wave equation. The method is based on an iterative descent method of first order which requires at each iteration the computation of a HUM control $v_{\omega}$ and the computation of a descent direction $\boldsymbol{\theta}$. The function $v_{\omega}$ is obtained using very efficient and robust schemes mixing gradient conjugate algorithm and finite difference scheme. The field $\boldsymbol{\theta}$, expressed independently of any adjoint control function, is obtained directly from $v_{\omega}$. These two aspects explain the interest of the approach we suggest. In addition, the numerical experiments illustrates the potential of the topological derivative to initialize the algorithm. It is interesting to notice that the method is able to find the HUM control distributed on $\omega$ (of arbitrarily small measure $|\omega|$ ) which drives to rest the system (1) after an arbitrarily small time $T$. For small values of $T$, the optimal domain is composed of several disjoints components distributed on the whole domain $\Omega$ so that all the wave components be observed by $\omega$ in the time lower or equal than $T$. Furthermore, from the numerical resolution of the convexified problem $\left(\mathcal{C P}_{\omega}\right)$ of $\left(\mathcal{P}_{\omega}\right)$, we may conjecture that the original problem is well-posed in the class of characteristic functions. Following [16] in a similar context, it is worth to investigate theoretically whether or not $(\mathcal{C P} \omega)$ and $\left(\mathcal{P}_{\omega}\right)$ always coincide for any initial condition in $H_{0}^{1}(\Omega) \times L^{2}(\Omega)$. It would be also interesting to extend this work to the 2-D case for more generals system like the elasticity one, and also to treat the boundary case. Finally, the treatment of time-dependent domains $(\omega(t))_{t \in[0, T]}$ seems open and challenging.

\section{Appendix}

\subsection{Sketch of the proof of Theorem 2.2}

The control $v_{\omega}$ of minimal $L^{2}$-norm is completely characterized by the couple $(\phi, \psi)$ given by (4)-(7). Let us apply the change of variables in the formulation of $\phi$ and $\psi$ and introduce the functions $\left(\phi^{\eta}(\boldsymbol{x}), \psi^{\eta}(\boldsymbol{x})\right)=\left(\phi\left(\boldsymbol{x}^{\eta}\right), \psi\left(\boldsymbol{x}^{\eta}\right)\right)$. According to the relations

$$
\left\{\begin{array}{l}
d x^{\eta}=\operatorname{det}\left(\nabla \mathcal{F}^{\eta}\right) d x=(1+\eta \operatorname{div} \boldsymbol{\theta}) d x, \\
\frac{\partial}{\partial x^{\eta}}=\frac{\partial}{\partial x^{\eta}} \cdot(I+\eta \nabla \boldsymbol{\theta})^{-1}=\frac{\partial}{\partial x^{\eta}} \cdot(I-\eta \nabla \boldsymbol{\theta})+O\left(\eta^{2}\right)
\end{array}\right.
$$


usual computations leads to the formulations :

$$
\begin{cases}\phi_{t t}^{\eta}-\operatorname{det}\left(\nabla \mathcal{F}^{\eta}\right)^{-1} \operatorname{div}\left(\mathcal{A}^{\eta}(\boldsymbol{\theta}) . \nabla \phi^{\eta}\right)=0, & \Omega \times(0, T), \\ \phi^{\eta}=0, & \partial \Omega \times(0, T), \\ \left(\phi^{\eta}(\cdot, 0), \phi_{t}^{\eta}(\cdot, 0)\right)=\left(\phi^{\eta 0}, \phi^{\eta 1}\right), & \Omega,\end{cases}
$$

and

$$
\begin{cases}\psi_{t t}^{\eta}-\operatorname{det}\left(\nabla \mathcal{F}^{\eta}\right)^{-1} \operatorname{div}\left(\mathcal{A}^{\eta}(\boldsymbol{\theta}) . \nabla \psi^{\eta}\right)=-\mathcal{X}_{\omega} \phi^{\eta}, & \Omega \times(0, T), \\ \psi^{\eta}=0, & \partial \Omega \times(0, T), \\ \left(\psi^{\eta}(\cdot, T), \psi_{t}^{\eta}(\cdot, T)\right)=(\mathbf{0}, \mathbf{0}), & \Omega,\end{cases}
$$

where $\mathcal{A}^{\eta}(\boldsymbol{\theta})=\operatorname{det}\left(\nabla \mathcal{F}^{\eta}\right)(I d+\eta \nabla \boldsymbol{\theta})^{-1} \cdot(I d+\eta \nabla \boldsymbol{\theta})^{-T}$. These two systems are a regular perturbation of (4) and (7) with respect to $\eta$. In particular, the term $\operatorname{div}\left(\mathcal{A}^{\eta} v\right)=\Delta v+\eta O\left(\operatorname{div}\left(\nabla v, \boldsymbol{\theta}, \nabla \boldsymbol{\theta}, \nabla^{2} \boldsymbol{\theta}, \ldots\right)\right)$ is regular perturbation of $\Delta v$ in the sense that the additional terms in $\eta$ does not contain derivatives of order higher than 2 in $v$ and that $\boldsymbol{\theta}$ is as regular as needed. Consequently, applying the HUM method, we obtain (proceeding as in [13]) that for all $\eta>0$ in the neighborhood of 0 , there exists an initial condition $\left(\phi^{0 \eta}, \phi^{1 \eta}\right) \in L^{2}(\Omega) \times H^{-1}(\Omega)$ such that $\left(\psi^{\eta}(\cdot, 0), \psi_{t}(\cdot, 0)\right)=$ $\left(y^{0}+\eta \nabla y^{0} \cdot \boldsymbol{\theta}, y^{1}+\eta \nabla y^{1} \cdot \boldsymbol{\theta}\right) \in H_{0}^{1}(\Omega) \times L^{2}(\Omega)$. We have truncated to the first order the Taylor expansion of $y^{0}(\boldsymbol{x}+\eta \boldsymbol{\theta}(\boldsymbol{x}))$ in order to avoid more regularity on $y^{0}$ and $y^{1}$. This keep unchanged the first derivative of $J$. Consequently, the function $-\mathcal{X}_{\omega} \phi^{\eta}$ associated to $\left(\phi^{0 \eta}, \phi^{1 \eta}\right)$ is the control of minimal $L^{2}$-norm of the system

$$
\begin{cases}y_{t t}^{\eta}-\operatorname{det}\left(\nabla \mathcal{F}^{\eta}\right)^{-1} \operatorname{div}\left(\mathcal{A}^{\eta}(\boldsymbol{\theta}) \cdot \nabla y^{\eta}\right)=v^{\eta} \mathcal{X}_{\omega}, & \Omega \times(0, T), \\ y^{\eta}=0, & \partial \Omega \times(0, T), \\ \left(y^{\eta}(\cdot, 0), y_{t}^{\eta}(\cdot, 0)\right)=\left(y^{0}+\eta \nabla y^{0} \cdot \boldsymbol{\theta}, y^{1}+\nabla y^{1} \cdot \boldsymbol{\theta}\right), & \Omega,\end{cases}
$$

Similarly, the function $-\left(\phi^{\eta}-\phi\right) \mathcal{X}_{\omega}$ associated to the initial condition $\left(\phi^{0 \eta}-\phi^{0}, \phi^{1 \eta}-\right.$ $\left.\phi^{1}\right)$ is the HUM control for the system

$$
\begin{cases}\left(y^{\eta}-y\right)_{t t}-\Delta\left(y^{\eta}-y\right)+\eta F\left(y^{\eta}, \boldsymbol{\theta}\right)=\left(v^{\eta}-v\right) \mathcal{X}_{\omega}, & \Omega \times(0, T), \\ y^{\eta}-y=0, & \partial \Omega \times(0, T), \\ \left(\left(y^{\eta}-y\right)(\cdot, 0),\left(y^{\eta}-y\right)_{t}(\cdot, 0)\right)=\eta\left(\nabla y^{0} \cdot \boldsymbol{\theta}, \nabla y^{1} \cdot \boldsymbol{\theta}\right), & \Omega,\end{cases}
$$

where $F$ is a function of $\mathcal{A}^{\eta}$, such that $\left(\left(y^{\eta}-y\right)(\cdot, T),\left(y^{\eta}-y\right)_{t}(\cdot, T)\right)=(\mathbf{0}, \mathbf{0})$. Then, by continuity of the solution and the control with respect to the initial condition, we conclude that there exist a function $\phi^{1}$ and $\psi^{1}$ such that for all $\eta>0, \phi^{\eta}-\phi=$ $\eta \phi^{1}+o(\eta)$ and $\psi^{\eta}-\psi=\eta \psi^{1}+o(\eta)$. $\phi^{1}$ and $\psi^{1}$ designates the first Lagrangian derivatives of $\phi$ and $\psi$ and fulfils the systems

$$
\begin{cases}\phi_{t t}^{1}-\Delta \phi^{1}-\nabla(\operatorname{div} \boldsymbol{\theta}) \cdot \nabla \phi+\operatorname{div}\left(\left(\nabla \boldsymbol{\theta}+\nabla \boldsymbol{\theta}^{T}\right) \cdot \nabla \phi\right)=0, & \Omega \times(0, T), \\ \phi^{1}=0, & \partial \Omega \times(0, T), \\ \left(\phi^{1}(\cdot, 0), \phi_{t}^{1}(\cdot, 0)\right)=\left(\phi^{1,0}, \phi^{1,1}\right), & \Omega,\end{cases}
$$

and

(60)

$$
\begin{cases}\psi_{t t}^{1}-\Delta \psi^{1}-\nabla(\operatorname{div} \boldsymbol{\theta}) . \nabla \psi+\operatorname{div}\left(\left(\nabla \boldsymbol{\theta}+\nabla \boldsymbol{\theta}^{T}\right) . \nabla \psi\right)=-\phi^{1} \mathcal{X}_{\omega} & \Omega \times(0, T), \\ \psi^{1}=0, & \partial \Omega \times(0, T), \\ \left(\psi^{1}(\cdot, T), \psi_{t}^{1}(\cdot, T)\right)=(\mathbf{0}, \mathbf{0}), & \Omega,\end{cases}
$$


such that $\left(\psi^{1}(\cdot, 0), \psi_{t}^{1}(\cdot, 0)\right)=\left(\nabla y^{0} \cdot \boldsymbol{\theta}, \nabla y^{1} \cdot \boldsymbol{\theta}\right) .-\phi^{1} \mathcal{X}_{\omega}$ is then the control of minimal $L^{2}$-norm supported on $\omega$ for the system (12). The existence of the control $v^{1}$ implies the existence of the first lagrangian shape derivative and permits to write

$$
\left.J\left(\mathcal{X}_{(I d+\eta \boldsymbol{\theta})(\omega)}\right)=\frac{1}{2} \int_{\omega} \int_{0}^{T}\left(v+\eta v^{1}\right)\right)^{2}(1+\eta \operatorname{div} \boldsymbol{\theta}) d t d x+o\left(\eta^{2}\right)
$$

Then, by passing to the limit according to the definition 2.1, we obtain the relation (11).

\subsection{Semi-discrete in space resolution of (6)}

The aim of this appendix is to detail the exact resolution in time of the semi-discrete wave systems in $\phi(4)$ and $\psi(7)$ which appear at each iteration of the conjugate gradient algorithm used to solve the linear system (6) (accordingly to the HUM method). The wave equations (4) and (7) are solved using a viscous finite difference scheme similar to (37). Let us first present the exact resolution in time of the semi-discrete system associated to (4):

$$
\left\{\begin{array}{lr}
\left(I+\frac{h^{2}}{4} \boldsymbol{\Delta}_{\boldsymbol{h}}\right)\left(\boldsymbol{\phi}_{\boldsymbol{h}}\right)_{t t}-\boldsymbol{\Delta}_{\boldsymbol{h}} \boldsymbol{\phi}_{\boldsymbol{h}}=0, & \Omega \times(0, T), \\
\boldsymbol{\phi}_{\boldsymbol{h}}=0, & \partial \Omega \times(0, T), \\
\left(\phi_{\boldsymbol{h}}(0),\left(\boldsymbol{\phi}_{\boldsymbol{h}}\right)_{t}(0)=\left(\boldsymbol{\phi}_{\boldsymbol{h}}^{\mathbf{0}}, \boldsymbol{\phi}_{\boldsymbol{h}}^{\mathbf{1}}\right),\right. & \Omega,
\end{array}\right.
$$

that we may write in the following vectorial form :

$$
\left\{\begin{array}{l}
M \phi_{\boldsymbol{h}}{ }^{\prime \prime}(t)+K_{h} \phi_{\boldsymbol{h}}(t)=0, \quad t \in(0, T), \\
\left(\phi_{\boldsymbol{h}}(0), \boldsymbol{\phi}_{\boldsymbol{h}}^{\prime}(0)\right)=\left(\boldsymbol{\phi}_{\boldsymbol{h}}^{\mathbf{0}}, \boldsymbol{\phi}_{\boldsymbol{h}}^{\mathbf{1}}\right)
\end{array}\right.
$$

where $M$ and $K_{h} \in \mathcal{M}_{N \times N}(\mathbb{R})$ designates the mass and stiffness matrices respectively. Theses two matrices are diagonal, symmetric and definite positive. Moreover, let us designates by $V_{h}, D_{h} \in \mathcal{M}_{N \times N}(\mathbb{R})$ the eigenvectors matrix and eigenvalues matrix respectively of $M^{-1} K_{h}$ such that

$$
K_{h} V_{h}=M V_{h} D_{h} .
$$

The matrix $D_{h}=\left(\lambda_{k}\right)_{1 \leq k \leq N}$ is diagonal, with $\lambda_{k}=4 h^{-2} \tan ^{2}(k \pi h / 2)$ whereas the matrix $V_{h}$ is symmetric and orthonormal such that $V_{h}^{2}=I_{N \times N}$. The eigenvector corresponding to $\lambda_{k}$ is $V_{k}=\left(V_{i k}\right)_{i=1, \ldots, N} \in \mathcal{M}_{N \times 1}(\mathbb{R})$, with $\left.V_{i k}=\sin (i k \pi h)\right)$. Therefore, the solution $\phi_{\boldsymbol{h}}(t)=\left(\phi_{i}(t)\right)_{(1 \leq i \leq N)}$ is

$$
\phi_{i}(t)=\sum_{k=1}^{N} V_{i k}\left(a_{k} \cos \left(\sqrt{\lambda_{k}} t\right)+\frac{b_{k}}{\sqrt{\lambda_{k}}} \sin \left(\sqrt{\lambda_{k}} t\right)\right), \quad 1 \leq i \leq N, t \geq 0
$$

where $\left(\boldsymbol{a}_{\boldsymbol{h}}, \boldsymbol{b}_{\boldsymbol{h}}\right)=\left(V_{h}^{-1} \boldsymbol{\phi}_{\boldsymbol{h}}^{\mathbf{0}}, V_{h}^{-1} \boldsymbol{\phi}_{\boldsymbol{h}}^{\mathbf{1}}\right)$ and $\boldsymbol{a}_{\boldsymbol{h}}=\left(a_{k}\right)_{(1 \leq k \leq N)}, \boldsymbol{b}_{\boldsymbol{h}}=\left(b_{k}\right)_{(1 \leq k \leq N)}$.

Similarly, the semi-discretization of (7) takes the following form

$$
\left\{\begin{array}{l}
M\left(\boldsymbol{\psi}_{\boldsymbol{h}}\right)_{t t}(t)+K_{h} \boldsymbol{\psi}_{\boldsymbol{h}}(t)=-\mathcal{X}_{\omega_{h}} \boldsymbol{\phi}_{\boldsymbol{h}}(t), \quad t \in(0, T) \\
\left(\boldsymbol{\psi}_{\boldsymbol{h}}(T), \boldsymbol{\psi}_{\boldsymbol{h}}^{\prime}(T)\right)=(\mathbf{0}, \mathbf{0}) .
\end{array}\right.
$$


Let $A$ be the diagonal matrix in $\mathcal{M}_{N \times N}$ associated to $-\mathcal{X}_{\omega_{h}}$. It follows that the new variable $\boldsymbol{z}_{\boldsymbol{h}}(t)=V_{h}^{-1} \boldsymbol{\psi}_{\boldsymbol{h}}(t)$ is solution of

$$
\left\{\begin{array}{l}
\boldsymbol{z}_{\boldsymbol{h}}^{\prime \prime}(t)+D_{h} \boldsymbol{z}_{\boldsymbol{h}}(t)=\left(M V_{h}\right)^{-1} A \phi_{\boldsymbol{h}}(t), \quad t \in(0, T) \\
\left(\boldsymbol{z}_{\boldsymbol{h}}(T), \boldsymbol{z}_{\boldsymbol{h}}^{\prime}(T)\right)=(\mathbf{0}, \mathbf{0})
\end{array}\right.
$$

Then, introducing the matrix $P=\left(M V_{h}\right)^{-1}$, the $j$-th component of the vector $z_{\boldsymbol{h}}$ is solution of

$$
z_{j}^{\prime \prime}(t)+\lambda_{j} z_{j}(t)=\left(P A \phi_{\boldsymbol{h}}(t)\right)_{j}, \quad t \geq 0, \quad 1 \leq j \leq N .
$$

Using (65), the right hand term is

$$
\begin{aligned}
\left(P A \phi_{\boldsymbol{h}}(t)\right)_{j} & =\sum_{1 \leq k, l \leq N} P_{j k} A_{k l} \phi_{l}(t) \\
& =\sum_{1 \leq k, l, p \leq N} P_{j k} A_{k l} V_{l p}\left(a_{p} \cos \left(\sqrt{\lambda_{p}} t\right)+\frac{b_{p}}{\sqrt{\lambda_{p}}} \sin \left(\sqrt{\lambda_{p}} t\right)\right)
\end{aligned}
$$

Consequently, the component $z_{j}$ is solution of the following system

$$
z_{j}^{\prime \prime}(t)+\lambda_{j} z_{j}(t)=\sum_{1 \leq p \leq N} \mathcal{B}_{j p}\left(a_{p} \cos \left(\sqrt{\lambda_{p}} t\right)+\frac{b_{p}}{\sqrt{\lambda_{p}}} \sin \left(\sqrt{\lambda_{p}} t\right)\right), \quad 1 \leq j \leq N
$$

where $\mathcal{B}=P A^{1} V_{h}$. Some computations lead to

$$
\begin{aligned}
z_{j}(t)= & C_{1}^{j} \cos \left(\sqrt{\lambda_{j}} t\right)+C_{2}^{j} \sin \left(\sqrt{\lambda_{j}} t\right) \\
& +\sum_{1 \leq p \leq N, \lambda_{p} \neq \lambda_{j}} \frac{\mathcal{B}_{j p}}{\lambda_{j}-\lambda_{p}}\left(a_{p} \cos \left(\sqrt{\lambda_{p}} t\right)+\frac{b_{p}}{\sqrt{\lambda_{p}}} \sin \left(\sqrt{\lambda_{p}} t\right)\right) \\
& +\frac{1}{2} \sum_{1 \leq p \leq N, \lambda_{p}=\lambda_{j}} \frac{\mathcal{B}_{j p}}{\lambda_{p}}\left(\left(a_{p}-b_{p} t\right) \cos \left(\sqrt{\lambda_{p}} t\right)+a_{p} \sqrt{\lambda_{p}} t \sin \left(\sqrt{\lambda_{p}} t\right)\right) \\
& \equiv C_{1}^{j} \cos \left(\sqrt{\lambda_{j}} t\right)+C_{2}^{j} \sin \left(\sqrt{\lambda_{j}} t\right)+F_{j}(t) .
\end{aligned}
$$

We then use the condition at time $T$ in (67) to fixe the constants $C_{1}^{j}$ and $C_{2}^{j}$, for $1 \leq j \leq N$;

$C_{1}^{j}=-\cos \left(\sqrt{\lambda_{j}} T\right) F_{j}(T)+\frac{\sin \left(\sqrt{\lambda_{j}} T\right)}{\sqrt{\lambda_{j}}} F_{j}^{\prime}(T), \quad C_{2}^{j}=-\sin \left(\sqrt{\lambda_{j}} T\right) F_{j}(T)-\frac{\cos \left(\sqrt{\lambda_{j}} T\right)}{\sqrt{\lambda_{j}}} F_{j}^{\prime}(T)$.

Finally, we obtain the vector $\boldsymbol{\psi}_{\boldsymbol{h}}(t)=V_{\boldsymbol{h}} \boldsymbol{z}_{\boldsymbol{h}}(t)$ for all $t \in(0, T)$.

Then, making $t=0$ in (71), we obtain

$$
z_{j}(0)=C_{1}^{j}+\sum_{1 \leq p \leq N, \lambda_{p} \neq \lambda_{j}} \frac{\mathcal{B}_{j p}}{\lambda_{j}-\lambda_{p}} a_{p}+\frac{1}{2} \sum_{1 \leq p \leq N, \lambda_{p}=\lambda_{j}} \frac{\mathcal{B}_{j p}}{\lambda_{p}} a_{p}
$$

and a similar relation for $z_{j}^{\prime}(0)$. This provides the explicit linear relation between the initial condition $\left(\boldsymbol{\phi}_{\boldsymbol{h}}^{\mathbf{0}}, \boldsymbol{\phi}_{\boldsymbol{h}}^{\mathbf{1}}\right)=\left(V_{h} \boldsymbol{a}_{\boldsymbol{h}}, V_{h} \boldsymbol{b}_{\boldsymbol{h}}\right)$ and $\left(\boldsymbol{\psi}_{\boldsymbol{h}}(0), \boldsymbol{\psi}_{\boldsymbol{h}}^{\prime}(0)\right)=\left(V_{h} \boldsymbol{z}_{\boldsymbol{h}}(0), V_{\boldsymbol{h}} \boldsymbol{z}_{\boldsymbol{h}}^{\prime}(0)\right)=$ $\left(\boldsymbol{y}_{\boldsymbol{h}}^{\mathbf{0}}, \boldsymbol{y}_{\boldsymbol{h}}^{\mathbf{1}}\right)$. The resulted linear system $\boldsymbol{\Lambda}_{\boldsymbol{h}}\left(\boldsymbol{\phi}_{\boldsymbol{h}}^{\mathbf{0}}, \boldsymbol{\phi}_{\boldsymbol{h}}^{\mathbf{1}}\right):=\left(\boldsymbol{y}_{\boldsymbol{h}}^{\mathbf{0}}, \boldsymbol{y}_{\boldsymbol{h}}^{\mathbf{1}}\right)$ is efficiently solved using a gradient conjugate algorithm initialized by $\left(\phi_{h}^{0}, \phi_{h}^{\mathbf{1}}\right)=(\mathbf{0}, \mathbf{0})$. 
Remark 4 The computation of $P=\left(M V_{h}\right)^{-1}$ is straightforward. From $M^{-1}=$ $V_{h} D_{M} V_{h}^{-1}$ and $V_{h}^{-1}=V_{h}$, the matrix $P$ is simply $P=\left(D_{M}\right)^{-1} V_{h}$ where $D_{M}$ is the diagonal matrix composed of the (analytically known) eigenvalues of $M$.

\section{References}

[1] Allaire G., Jouve F., Toader A.M., Structural optimization using sensitivity analysis and level-set methods, J. Comp. Phys. 194(1), 363-393 (2004).

[2] Asch M., Lebeau G., Geometrical aspects of exact controllability for the wave equation - A numerical study, Esaim : Cocv, 3, 163-212 (1998).

[3] Barles G., Sougadinis P.E., On the large time behavior of solutions of Hamilton-Jacobi equations, SIAM J. Math. Anal., 31(4), 925-939 (2000).

[4] Burger M., Osher S.J., A survey on level set methods for inverse problems and optimal design, European Journal of Applied Mathematics, 16(2), 263-301 (2005).

[5] Cagnol J., Zolesio J.P., Shape derivative in the wave equation with Dirichlet boundary condition, J. Diff. Eq 158, 175-210, (1999).

[6] Castro C., Micu S., Münch A., Numerical approximation of the boundary control of the 2-D wave equation with mixed finite elements., Submitted to IMA Numerical Analysis.

[7] Cea J., Conception optimale ou identification de formes, calcul rapide de la dérivée directionnelle de la fonction coût, Math. Model. Num. Anal., 20(3), 371-402 (1986).

[8] Delfour M.C., Zolesio J.P., Shapes and Geometries - Analysis, Differential Calculus and Optimization, Advances in Design and Control, Siam, (2001).

[9] Garreau S., Guillaume P., Masmoudi M., The topological asymptotic for PDE systems : the elasticity case, SIAM J. Control Optim, 39(6), 1756-1778 (2001).

[10] Glowinski R., Li C.H., Lions J-L., A numerical approach to the exact boundary controllability of the wave equation (I). Dirichlet controls: Description of the numerical methods, Int. J. Numer. Methods. Eng., 27(3), 623-636 (1989).

[11] Hebrard P., Henrot A., Optimal shape and position of the actuators for the stabilization of a string, Systems and control letters, 48, 199-209 (2003).

[12] Henrot A., Pierre M., Variation et optimisation de formes : une analyse géométrique, Mathématiques et Applications 48, Springer, (2005).

[13] Lions J.L., Contrôlabilité exacte, stabilisation et perturbations de systèmes distribués, Tome 1, Masson, RMA 8, Paris (1988).

[14] Münch A., A uniformly controllable and implicit scheme for the 1-D wave equation, Mathematical Modelling and Numerical Analysis 39(2), 377-418, (2005).

[15] Münch A., Optimal internal stabilization of a damped wave equation by a level set approach, Prépublication du laboratoire de Mathématiques de Besancon, 2005/1. (downlable at www-math.univ-fcomte.fr/research.htm). 
[16] Münch A., Pedregal P., Periago F., Relaxation of an optimal shape design problem for the wave equation, Prépublication du laboratoire de Mathématiques de Besancon, 2006/06. (downlable at www-math.univ-fcomte.fr/research.htm).

[17] Sokolowski J., Zochowski A., On the topological derivative in shape optimization, SIAM J. Control Optim., 37(4), 1251-1272 (1999).

[18] Osher S.J., Fedkiw R., Level set methods and dynamics implicit surfaces, Applied Mathematics Sciences, Springer-Verlag, 153, (2002).

[19] Sethian J.A., Level set methods: Evolving interfaces in geometry, fluid mechanics, computer vision, and materials science, Cambridge University Press, (1996).

[20] Wang M.Y., Wang X., Guo D., A level set method for structural topology optimization, Computat. Methods. Appl. Mech. Engrg. 192, 227-246, (2003).

[21] Zuazua E., Propagation, Observation, Control and Numerical Approximation of Waves approximated by finite difference methods, SIAM Review, 47(2), 197-243, (2005). 